\title{
Time-Variant Doppler PDFs and Characteristic Functions for the Vehicle-to-Vehicle Channel
}

\author{
Michael Walter, Member, IEEE, Dmitriy Shutin, Senior Member, IEEE, Armin Dammann, Member, IEEE
}

\begin{abstract}
Non-stationarity of vehicle-to-vehicle channels is one of the key elements that has to be taken into account for accurate channel modeling. The time-variance and its dual - the frequency selectivity - lead to non-stationarity. These can be assessed by both the temporal autocorrelation function and the Doppler spectrum, respectively. For fixed-to-mobile channels closed-form solutions for autocorrelation functions and Doppler spectra are well known. For vehicle-to-vehicle channels closed-form solutions exist, if uncorrelated double-bounce scattering is assumed. For time-variant, delay-dependent, correlated single-bounce scattering expressions are yet to be found. This contribution addresses the mentioned problem. Specifically, the proportionality between the Doppler probability density function (pdf) and Doppler power spectral density in time-varying scenarios for non-stationary, uncorrelated scattering is demonstrated. The latter also implies a proportionality between the characteristic function and the corresponding autocorrelation function; these functions are the Fourier transforms of the Doppler pdf and Doppler power spectral density, respectively. It is shown that time-varying characteristic functions and Doppler pdfs for general vehicle-tovehicle scenarios can be derived in prolate spheroidal coordinates. The investigation of the Doppler frequency in these coordinates allows us to derive expressions of the maximum and minimum frequencies of the Doppler pdf in the vicinity of line-of-sight. Several vehicular scenarios of interest are investigated and closedform solutions for the Doppler pdf and characteristic function are presented. An analysis of the results shows that the obtained expressions generalize well the known closed-form results for stationary channels. This further permits deriving some timevariant statistical channel parameters like mean Doppler and Doppler spread. These parameters are particularly important when designing a Wiener filter or estimating propagation channel characteristics for highly time-variant vehicle-to-vehicle channels.
\end{abstract}

Index Terms-Characteristic function, Doppler pdf, vehicleto-vehicle channel, geometry-based stochastic channel modeling, prolate spheroidal coordinates.

\section{INTRODUCTION}

$\mathbf{V}$ EHICLE-TO-VEHICLE communication is a rapidly developing communications technology that promises to revolutionize the ever-growing vehicular traffic handling and to increase the safety of the road users [1]. Vehicles are envisioned to be able to exchange sensor data and thus obtain important information about their surroundings. Such technologies ultimately rely on wireless communications, and specifically on mobile-to-mobile communication systems. Their development and implementation requires understanding of the vehicular propagation channel; the latter, in contrast to fixed-to-mobile systems, is known to be more complicated.

For narrowband, fixed-to-mobile channels, purely statistical modeling approaches like in [2] and [3] are sufficient to accurately represent the channel. These are based on the widesense stationary uncorrelated scattering (WSSUS) assumption, introduced by Bello in [4]. Clarke derived the Doppler power spectrum for uniformly distributed scatterers in [2], which is known today as Jakes Doppler spectrum. The autocorrelation function of the stochastic process described by the Jakes spectrum can be computed through the inverse Fourier transform of the Doppler power spectrum, which is known to result in a Bessel function. These functions are used to stochastically simulate channel realizations and thus test new communication systems.

For mobile-to-mobile (M2M) channels, however, the situation is different, since both transmitter and receiver are mobile. As a consequence, the channel statistics are not necessarily constant over time and channels possibly violate the WSSUS assumption according to [5] and [6]. Therefore, new models that take the non-stationarity into account have to be developed.

The local scattering function, proposed in [7] is a theoretical model that describes non-WSSUS channels. It can be seen as generalization of the Bello model. One of the first stochastic models for the vehicle-to-vehicle (V2V) channel, which is a class of M2M channels, was presented in [8]. It has been shown that the Doppler spectrum for multiple-bounce scattering, assuming the scattering processes at transmitter and receiver are uncorrelated, can be obtained in form of the elliptic integral of the first kind, which results from a convolution of two Jakes spectra. The corresponding autocorrelation is therefore a multiplication of two Bessel functions [8]. By assuming a stationary scatterer and both moving transmitter (TX) and receiver (RX), a multiple bounce model with uniformly distributed scatterers around TX and RX can be transformed into an equivalent two-ring [9], i.e., for 2D based on the work of [10] or a two-cylinder model [11], i.e., for 3D scenarios, with scatterers uniformly distributed on circles or cylinders. In addition to the temporal autocorrelation the authors in [12] define a spatial correlation function for Multiple Input Multiple Output (MIMO) scenarios using results form [13]. Subsequently, this model was first extended to a MIMO model with non-uniform scattering in [14], later to a threedimensional MIMO model in [15], and finally, to a wideband MIMO in [16]. In contrast to purely stochastic models, these modeling approaches are known as geometric-stochastic modeling, where the geometry of the vehicle constellation and their environment is taken into account. A further extension to a non-stationary modeling technique is presented for example in [17] and [18]. Moving scatterers further complicate the model and are studied in [19] and [20], where the authors derive the autocorrelation function, power spectral density, and the Doppler spread. 
The mentioned models, however, use temporal averages of channel characteristics to derive statistical properties of the corresponding stochastic process. This implicitly implies ergodicity of the observed process; as such, the temporal average can be used instead of ensemble average of the process. Note that a strict-sense ergodic process is always stationary according to [21]. Yet, because of the time-variance, the ergodicity assumption is only valid over relatively short time intervals. In practice, such time intervals are often scenariodependent. Moreover, the ergodicity necessitates stationarity, which is generally violated in $\mathrm{V} 2 \mathrm{~V}$ channels according to [6]. In our description, we use a time-variant ensemble average to characterize the statistical properties of the stochastic process, which circumvents the above mentioned shortcomings. This is done by using time-variant, delay-dependent Doppler pdfs derived by postulating the proportionality between the pdf and an equivalent Doppler power spectral density [22]. There, the delay-dependent Doppler pdf is obtained in Cartesian coordinates and contains quite unyielding mathematical expressions, which made further analysis rather difficult. Also, the proportionality assumption between the derived pdfs and the Doppler spectral density was presumed and so far has only been experimentally verified in [23] and [24].

In this paper, we describe the delay-dependent Doppler pdf and its Fourier dual - the characteristic function - in a time-variant manner for single-bounce scattering ${ }^{1}$ based on the model presented in [22]. Both Doppler and characteristic function allow different insights into the time-variance of the channel. As we will show, by using a mathematical description of such channels in a prolate spheroidal coordinate system (PSCS) instead of Cartesian coordinates, it becomes possible to derive the Doppler pdf and the corresponding characteristic function for arbitrary, but fixed delays with respect to the scatterers. Moreover, the PSCS allows for a simpler delaydependent channel description. By using the new formulation, we obtain general closed-form solutions for the time-variant, delay-dependent characteristic functions and Doppler pdfs for the line-of-sight (LOS) and infinite delay for any velocity vector configuration. We show that the results generalize some expression already known from the literature. Additionally, we present general time-variant, delay-dependent mean Doppler and Doppler spread expressions. By investigating the presented equations, a common description for both the LOS and singlebounce scattering components can be found. We found that in the $\epsilon$-vicinity of the LOS delay, the width of the Doppler pdf experiences an abrupt increase. The frequencies that characterize the support of the resulting spectrum - the limiting frequencies - can also be derived in closed form. Thus, we present closed-form expressions for the time-variant limiting frequencies of the Doppler spectrum for the line-of-sight, in the $\epsilon$-vicinity of the line-of-sight and for infinite delay for any velocity configuration. These are the new results for $\mathrm{V} 2 \mathrm{~V}$ channels. Note that the scattering contribution close to the LOS delay typically has stronger power, and thus a larger impact on

\footnotetext{
${ }^{1}$ Double-bounce scattering is not in the scope of this work, yet our model can potentially be extended by using $k$-ellipses [25] in future publications.
}

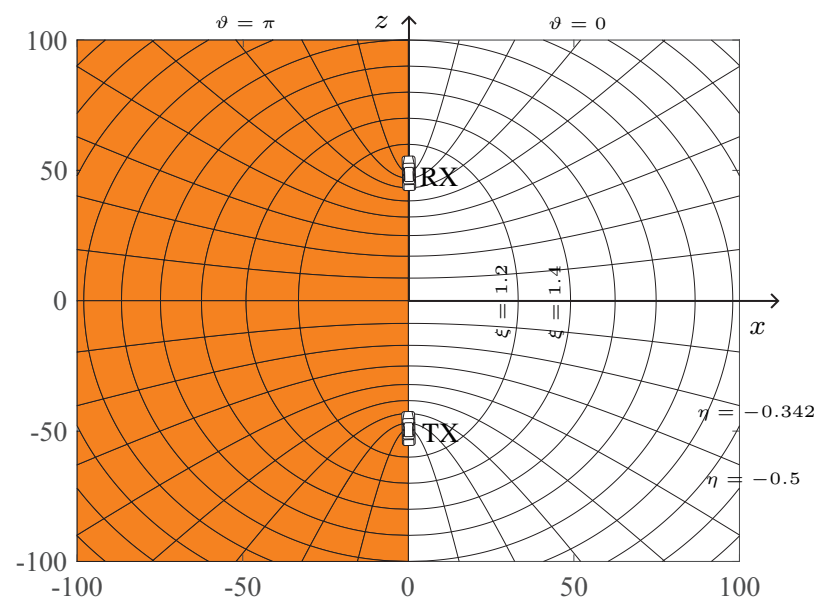

Fig. 1. Prolate spheroidal coordinate system with the transmitter and receiver in the foci of the ellipses and hyperbolas, which represent the lines of constant coordinates. $\xi \in[1.0,2.8]$ in steps of 0.2 and $\eta \in[-1,1]$ with a non-uniform step size. The half-planes are given by $\vartheta=0$ and $\vartheta=\pi$ (shaded). The relationship to the Cartesian coordinate system is shown.

the transmitted signal as compared to scattering components with longer delays.

In this paper, we also prove the proportionality between the channel characteristic function and the channel autocorrelation function, or, equivalently, the proportionality between the Doppler pdf and the Doppler power spectral density for nonstationary, uncorrelated scattering. This result generalizes that of Höher in [26] obtained for WSSUS channels.

The remainder of the paper is structured as follows. In Section II, we explain the use of the prolate spheroidal coordinate system and the coordinate transformation itself. Subsequently, the Doppler pdf and the corresponding characteristic function are derived in the new coordinate system and the proportionality to the Bello functions is demonstrated. An analysis of the the Doppler frequency in Section III provides closedform expressions for the limiting frequencies. The results for exemplary vehicular scenarios are presented in Section IV. Finally, conclusions are drawn in Section V.

\section{Prolate Spheroidal Coordinate System}

The mathematical tractability of the following analysis of $\mathrm{V} 2 \mathrm{~V}$ propagation channels is based on an appropriate coordinate system. As shown in [27], a suitable coordinate system for the V2V channel is the PSCS. In general, it is a three-dimensional, curvilinear, orthogonal coordinate system, which adequately describes two-center problems, exemplified well by the M2M channel with the transmitter and receiver forming the two centers, as shown in Fig. 1. The origin of the coordinate system is always located in the middle between the two vehicles and moves along with them. For the V2V channel the dimensionality naturally reduces from three to two later on. In Fig. 1, the third dimension is obtained by a rotation of the $x-z$ plane around the $z$-axis, which results in ellipsoidal and hyperboloid iso-surfaces. Note that the third orthogonal surface in the PSCS is formed by a half-plane, shown as shaded region in Fig. 1. 
The relationship between Cartesian and prolate spheroidal coordinates is according to [28] given by

$$
\begin{aligned}
& x=l \sqrt{\left(\xi^{2}-1\right)\left(1-\eta^{2}\right)} \cos (\vartheta) \\
& y=l \sqrt{\left(\xi^{2}-1\right)\left(1-\eta^{2}\right)} \sin (\vartheta) \\
& z=\quad l \xi \eta,
\end{aligned}
$$

with $l$ being the focus distance, i.e. the distance from the vehicles to the origin, $\xi \in[1, \infty)$, and $\eta \in[-1,1] . \xi=\frac{\tau}{\tau_{\text {los }}}$ corresponds to a normalized delay. This allows for a very general description of the Doppler frequency later on, where the absolute delay between transmitter and receiver does not matter anymore. The absolute delay, however, can be calculated by $\tau=\tau_{\operatorname{los}} \xi=\frac{2 l \xi}{c}$ with $c$ being the speed of light. Iso-surfaces of $\xi$ are prolate spheroidal ellipsoids, whereas constant values of $\eta$ produce hyperboloid surfaces. Geometrically, a fixed $\xi$-coordinate represents locations where the sum of the delays to the foci, i.e., vehicles, is constant. A fixed $\eta$-coordinate represents locations where the difference of the delays to the vehicles is constant, yet in contrast to $\xi$ we do not need to use this physical interpretation is our paper. As we will show, this coordinate transform effectively leads to polynomial expressions in the $\xi$ - and $\eta$-coordinates.

Let us point out that the big advantage of the PSCS is that by fixing the $\xi$-coordinate, we readily obtain a fixed delay. As such, our description of the delay-dependent Doppler pdf becomes only dependent on the other two coordinates in the PSCS; in a planar geometry case this even reduces to a single PSCS coordinate. In contrast to that, fixing the delay in a Cartesian coordinate system still implies the dependency on all spatial coordinates $x$ and $y$ and $z$, which makes the resulting expressions more difficult.

The description in the new coordinate system allows us to express both delay and Doppler frequency in a more compact notation in comparison to Cartesian coordinates. Specifically, the delay from transmitter to an arbitrary scatter $d_{\mathrm{t}}$ and the delay from this scatterer to the transmitter $d_{\mathrm{r}}$, can be defined as

$$
\begin{aligned}
d_{\mathrm{t}} & =(\xi+\eta) l, \\
d_{\mathrm{r}} & =(\xi-\eta) l, \\
d_{\mathrm{sc}} & =d_{\mathrm{t}}+d_{\mathrm{r}}=2 \xi l,
\end{aligned}
$$

where the total distance $d_{\mathrm{sc}}$, or the total delay $\tau_{\mathrm{sc}}=\frac{d_{\mathrm{sc}}}{c}$, depends only on the $\xi$-coordinate. As has been shown by the authors in [22], the Doppler frequency can be calculated as spatial gradient of the transmitter and receiver distances $d_{\mathrm{t}}$ and $d_{\mathrm{r}}$ projected onto their velocity vectors. This results in

$$
f_{\mathrm{d}}(\mathbf{x})=\left(\mathbf{v}_{\mathrm{t}}^{\mathrm{T}} \nabla d_{\mathrm{t}}(\mathbf{x})+\mathbf{v}_{\mathrm{r}}^{\mathrm{T}} \nabla d_{\mathrm{r}}(\mathbf{x})\right) \frac{f_{\mathrm{c}}}{c} .
$$

A delay-dependent representation of the Doppler frequency in Cartesian coordinates depends, however, on the $x-, y$-, and $z$ coordinate, so that the calculation of the Doppler pdf becomes very cumbersome. To circumvent this, we transform the mathematical analysis in the PSCS and express the gradient in (3) in prolate spheroidal coordinates.
Consider an arbitrary scalar function $\Psi(\xi, \eta, \vartheta)$ in the PSCS. Its gradient $\nabla \Psi$ can be computed as in [29] by

$$
\nabla \Psi(\xi, \eta, \vartheta)=\frac{1}{h_{\xi}} \frac{\partial \Psi}{\partial \xi} \mathbf{e}_{\xi}+\frac{1}{h_{\eta}} \frac{\partial \Psi}{\partial \eta} \mathbf{e}_{\eta}+\frac{1}{h_{\vartheta}} \frac{\partial \Psi}{\partial \vartheta} \mathbf{e}_{\vartheta}
$$

where $\mathbf{e}_{\xi}, \mathbf{e}_{\eta}$, and $\mathbf{e}_{\vartheta}$ are the orthonormal basis vectors of the PSCS. They are obtained by transforming the Cartesian basis vectors $\mathbf{e}_{\mathrm{x}}, \mathbf{e}_{\mathrm{y}}$ and $\mathbf{e}_{\mathrm{z}}$ using the following identity

$$
\mathbf{e}_{\alpha}=\frac{\partial x}{\partial \alpha} \frac{\mathbf{e}_{\mathrm{x}}}{h_{\alpha}}+\frac{\partial y}{\partial \alpha} \frac{\mathbf{e}_{\mathrm{y}}}{h_{\alpha}}+\frac{\partial z}{\partial \alpha} \frac{\mathbf{e}_{\mathrm{z}}}{h_{\alpha}},
$$

where the subscript $\alpha \in\{\xi, \eta, \vartheta\}$. In (4) and (5) the scalars $h_{\xi}, h_{\eta}$, and $h_{\vartheta}$ are the so called scale factors. They account for the normalization of the basis vectors in the transformed coordinate system to ensure orthonormality of the transformed basis. For orthogonal coordinates the scale factors are the roots of the three non-zero elements $h_{i}=\sqrt{g_{i i}}$ of the metric tensor g (see [29] for more details). In the PSCS, the scale factors are calculated as

$$
\begin{aligned}
& h_{\xi}=l \sqrt{\frac{\left(\xi^{2}-\eta^{2}\right)}{\left(\xi^{2}-1\right)}}, \quad h_{\eta}=l \sqrt{\frac{\left(\xi^{2}-\eta^{2}\right)}{\left(1-\eta^{2}\right)}}, \\
& h_{\vartheta}=l \sqrt{\left(\xi^{2}-1\right)\left(1-\eta^{2}\right)} .
\end{aligned}
$$

By transforming (3) into prolate spheroidal coordinates, the Doppler frequency can be expressed as

$$
\begin{aligned}
& f_{\mathrm{d}}(t ; \xi, \eta, \vartheta)=\frac{f_{\mathrm{c}}}{c}( \\
& \quad \frac{\sqrt{\left(\xi^{2}-1\right)\left(1-\eta^{2}\right)}}{\xi+\eta}\left(v_{\mathrm{t}}^{x} \cos \vartheta+v_{\mathrm{t}}^{y} \sin \vartheta\right)+\frac{\xi \eta+1}{\xi+\eta} v_{\mathrm{t}}^{z} \\
& \left.+\frac{\sqrt{\left(\xi^{2}-1\right)\left(1-\eta^{2}\right)}}{\xi-\eta}\left(v_{\mathrm{r}}^{x} \cos \vartheta+v_{\mathrm{r}}^{y} \sin \vartheta\right)+\frac{\xi \eta-1}{\xi-\eta} v_{\mathrm{r}}^{z}\right) \\
& =f_{\mathrm{d}, \mathrm{TX}}(t ; \xi, \eta, \vartheta)+f_{\mathrm{d}, \mathrm{RX}}(t ; \xi, \eta, \vartheta),
\end{aligned}
$$

where $f_{\mathrm{d}, \mathrm{TX}}(t ; \xi, \eta, \vartheta)$ consists of the first two summands in (7) and thus represents the contributions of the transmitter. Similarly, $f_{\mathrm{d}, \mathrm{RX}}(t ; \xi, \eta, \vartheta)$ consists of the last two summands in (7) and thus represents the contributions of the receiver. Note that due to the implicit time-variance of the velocity vectors, the Doppler frequency becomes time-variant which is noted by the variable $t$. In the following, we show how delaydependent Doppler pdfs and the corresponding characteristic functions are computed for V2V channels in the PSCS.

\section{A. Time-Variant, Delay-Dependent Doppler PDF}

In the subsequent analysis, we go from the general 3D M2M description to a 2D V2V description. This means that

$$
y=l \sqrt{\left(\xi^{2}-1\right)\left(1-\eta^{2}\right)} \sin (\vartheta)=0,
$$

which is true for $\vartheta=\{0, \pi\}$. The analysis relies on the use of a geometric-stochastic description of the vehicular environment to obtain a time-variant joint Doppler delay pdf $p\left(t ; f_{\mathrm{d}}, \xi\right)$. The joint Doppler delay pdf is obtained as $p\left(t ; f_{\mathrm{d}}, \xi\right)=p(t ; \xi) p\left(t ; f_{\mathrm{d}} \mid \xi\right)$. Please note that we represent the Doppler pdf as a function of normalized delay $\xi$ rather than absolute delay $\tau$. 
In the following, we study the delay-dependent conditional Doppler pdf $p\left(t ; f_{\mathrm{d}} \mid \xi\right)$ in the PSCS. The derivation is obtained by a coordinate transformation of the scatterer distribution $p(\eta \mid \xi)$ as was shown in [27]; subsequently, we will summarize the key steps in computing $p\left(t ; f_{\mathrm{d}} \mid \xi\right)$.

We model the delay-dependent pdf of uniformly distributed scatterers as shown in [22]. To this end, we fix the $\xi$ coordinate, thus making use of the specific property of the PSCS, and make an assumption that the scatterer distribution is independent of the absolute time $t$. Then we consider the parameter $\eta \in[-1,1]$ of the half-ellipse that specifies a scatterer lying on it. For a fixed $\xi$, it can be shown [22], [30] that the conditional pdf $p(\eta \mid \xi)$ can be computed by applying standard rules of probability density transformations [31] by

$$
\begin{aligned}
p(\eta \mid \xi) & =\frac{1}{2} p(\eta, \vartheta=0 \mid \xi)+\frac{1}{2} p(\eta, \vartheta=\pi \mid \xi) \\
& =\left.\frac{1}{4 E\left(\frac{1}{\xi^{2}}\right)} \sqrt{\frac{1-\frac{\eta^{2}}{\xi^{2}}}{1-\eta^{2}}}\right|_{\vartheta=0}+\left.\frac{1}{4 E\left(\frac{1}{\xi^{2}}\right)} \sqrt{\frac{1-\frac{\eta^{2}}{\xi^{2}}}{1-\eta^{2}}}\right|_{\vartheta=\pi}
\end{aligned}
$$

where $E\left(\frac{1}{\xi^{2}}\right):=\int_{0}^{1} \sqrt{\frac{1-\frac{\eta^{2}}{\xi^{2}}}{1-\eta^{2}}} \mathrm{~d} \eta$ is the complete elliptic integral of the second kind. Note that the pdf $p(\eta \mid \xi)$ could be simplified, since the summands are the same. Yet, we purposely keep the expression in this form, since the Doppler frequency can be different in both half-planes. This will become clear later, as we discuss the calculation of the Doppler pdf and the corresponding characteristic function.

Following [27], we compute a time-variant, delay-dependent Doppler pdf as

$$
\begin{aligned}
p\left(t ; f_{\mathrm{d}} \mid \xi\right) & =\left.\sum_{\eta^{\prime} \in\left\{\mathcal{F}^{-1}\left(f_{\mathrm{d}}\right)\right\}} \frac{1}{2} \frac{p(\eta, \vartheta=0 \mid \xi)}{\left|\frac{\partial f_{\mathrm{d}}(t ; \eta, \vartheta=0 \mid \xi)}{\partial \eta}\right|}\right|_{\eta=\eta^{\prime}} \\
& +\left.\sum_{\eta^{\prime} \in\left\{\mathcal{F}^{-1}\left(f_{\mathrm{d}}\right)\right\}} \frac{1}{2} \frac{p(\eta, \vartheta=\pi \mid \xi)}{\left|\frac{\partial f_{\mathrm{d}}(t ; \eta, \vartheta=\pi \mid \xi)}{\partial \eta}\right|}\right|_{\eta=\eta^{\prime}},
\end{aligned}
$$

with the Doppler frequency $f_{\mathrm{d}}(t ; \xi, \eta, \vartheta)$ being computed according to (7). The sum in (11) accounts for the fact that one Doppler frequency results possibly in several values of $\eta$; this is sometimes referred to as a multivalued function. A more detailed description of the transformation of the pdfs can be found in [22].

\section{B. Time-Variant, Delay-Dependent Characteristic Function}

The characteristic function is defined as the inverse Fourier transform of the probability density function, see, e.g., [21] and [31]. It thus gives an alternative description of a random variable described by a pdf. For instance, a characteristic function can be used to facilitate the computation of the moments of a random variable, or compute a distribution of a sum of independent random variables. In our case, by postulating the proportionality between the conditional Doppler pdf and corresponding power spectrum, and due to the properties of the Fourier transform, the characteristic function and the correlation function are likewise proportional.
In the following, we use the PSCS to calculate the characteristic function. To this end, we apply an inverse Fourier transform to the pdf $p(\eta \mid \xi)$ instead of using the Doppler pdf $p\left(t ; f_{\mathrm{d}} \mid \xi\right)$ for both half-planes $\vartheta=0$ and $\vartheta=\pi$. Thus, we obtain simpler derivations. A similar approach was also used in [21, Appendix A].

The characteristic function is calculated as

$$
\begin{aligned}
& \Phi(t ; u \mid \xi)= \\
& =\frac{1}{4 E\left(\frac{1}{\xi^{2}}\right)} \int_{-1}^{1} \sqrt{\frac{1-\frac{\eta^{2}}{\xi^{2}}}{1-\eta^{2}}} \exp \left(\mathrm{j} 2 \pi u f_{\mathrm{d}}(t ; \xi, \eta, 0)\right) \mathrm{d} \eta \\
& +\frac{1}{4 E\left(\frac{1}{\xi^{2}}\right)} \int_{-1}^{1} \sqrt{\frac{1-\frac{\eta^{2}}{\xi^{2}}}{1-\eta^{2}}} \exp \left(\mathrm{j} 2 \pi u f_{\mathrm{d}}(t ; \xi, \eta, \pi)\right) \mathrm{d} \eta .
\end{aligned}
$$

Note that $u:=\Delta t-$ an independent variable of the characteristic function - is equivalent to a time lag in a classical correlation function. As we will see later, closed-form expressions for (11) can be computed for some special cases.

The characteristic function - an equivalent representation of the channel correlation function - permits deriving other important statistical parameters that summarize the instantaneous dynamics of the channel. Specifically, we can determine the time-variant, delay-dependent, first and second central moments of the channel Doppler spread, which are known as the mean Doppler $\mu(t ; \xi)$ and the corresponding standard deviation $\sigma(t ; \xi)$. This is done by calculating the first and second derivative of the characteristic function at $u=0$.

The first and second derivative with respect to the coordinate $u$ of the characteristic function $\Phi(t ; u \mid \xi)$ are calculated to determine the mean and Doppler spread by [21] as

$$
\begin{aligned}
\mu(t ; \xi) & =\left.\frac{\frac{\partial}{\partial u} \Phi(t ; u \mid \xi)}{\mathrm{j} 2 \pi}\right|_{u=0}, \\
\sigma(t ; \xi) & =\left.\frac{\sqrt{\left(\frac{\partial}{\partial u} \Phi(t ; u \mid \xi)\right)^{2}-\frac{\partial^{2}}{\partial u^{2}} \Phi(t ; u \mid \xi)}}{2 \pi}\right|_{u=0} .
\end{aligned}
$$

The first derivative $\frac{\partial}{\partial u} \Phi(t ; u \mid \xi)$ can be computed by the following expression

$$
\begin{aligned}
& \quad \frac{\partial}{\partial u} \Phi(t ; u \mid \xi)=\frac{\mathrm{j} 2 \pi}{4 E\left(\frac{1}{\xi^{2}}\right)}( \\
& \int_{-1}^{1} \sqrt{\frac{1-\frac{\eta^{2}}{\xi^{2}}}{1-\eta^{2}}} f_{\mathrm{d}}(t ; \xi, \eta, 0) \exp \left(\mathrm{j} 2 \pi u f_{\mathrm{d}}(t ; \xi, \eta, 0)\right) \mathrm{d} \eta \\
& \left.+\int_{-1}^{1} \sqrt{\frac{1-\frac{\eta^{2}}{\xi^{2}}}{1-\eta^{2}}} f_{\mathrm{d}}(t ; \xi, \eta, \pi) \exp \left(\mathrm{j} 2 \pi u f_{\mathrm{d}}(t ; \xi, \eta, \pi)\right) \mathrm{d} \eta\right),
\end{aligned}
$$


and the second derivative $\frac{\partial^{2}}{\partial u^{2}} \Phi(t ; u \mid \xi)$ is similarly given as

$$
\begin{aligned}
& \frac{\partial^{2}}{\partial u^{2}} \Phi(t ; u \mid \xi)=\frac{-4 \pi^{2}}{4 E\left(\frac{1}{\xi^{2}}\right)}( \\
& \int_{-1}^{1} \sqrt{\frac{1-\frac{\eta^{2}}{\xi^{2}}}{1-\eta^{2}}} f_{\mathrm{d}}(t ; \xi, \eta, 0)^{2} \exp \left(\mathrm{j} 2 \pi u f_{\mathrm{d}}(t ; \xi, \eta, 0)\right) \mathrm{d} \eta \\
& \left.+\int_{-1}^{1} \sqrt{\frac{1-\frac{\eta^{2}}{\xi^{2}}}{1-\eta^{2}}} f_{\mathrm{d}}(t ; \xi, \eta, \pi)^{2} \exp \left(\mathrm{j} 2 \pi u f_{\mathrm{d}}(t ; \xi, \eta, \pi)\right) \mathrm{d} \eta\right) .
\end{aligned}
$$

Like the characteristic function, the mean Doppler $\mu(t ; \xi)$ and Doppler spread $\sigma(t ; \xi)$ can be calculated in closed-form expressions for some special cases. Let us stress that by using $\frac{\partial^{2}}{\partial u^{2}} \Phi(t ; u \mid \xi)$, it also becomes possible to calculate the delaydependent level crossing rate (LCR) and average duration of fades (ADF), provided a Rayleigh or Rice fading for the amplitude distribution is assumed.

\section{Proportionality between Doppler Probability Density Function and Doppler Power Spectral Density}

Our previous analysis is based on the assumption that $p\left(t ; f_{\mathrm{d}} \mid \xi\right)$ and the corresponding Doppler spectral density are proportional. It is known from [26] and [32] that for WSSUS channels the joint delay Doppler pdf $p\left(\tau, f_{\mathrm{d}}\right)$ is exactly proportional to the scattering function $P_{s}\left(\tau, f_{\mathrm{d}}\right)$. Similarly, for quasi wide-sense stationary uncorrelated scattering (QWSSUS) channels, this proportionality will hold for a limited period of time and a limited frequency range. In practice, it is common to avoid dealing with the non-stationarity of the channel by restricting the observation time. For instance, one can identify the quasi wide-sense stationary (QWSS) regions for $\mathrm{V} 2 \mathrm{~V}$ as in [33] or determine the local quasi-stationarity regions as shown in [34]. There are also statistical tests available to check the wide-sense stationary (WSS) assumption for MIMO channels [35]. Yet, these techniques still exploit the stationarity assumption to avoid challenges of time-variant channels.

Here, we relax the WSSUS case by assuming that only the uncorrelated scattering (US) property holds and show that the proportionality between the Doppler pdf and Doppler power spectral density can be established point-wise in time. This concurs with the statement that "the vehicular channel violates the WSS much stronger than the US assumption" in [6]. This approach thus better reflects the time-variant nature of the wireless channel. Our proof extends the WSSUS results in [26], [32] by taking the non-stationarity into account.

For a US stochastic process, it follows that the time-variant transfer function can be arbitrarily well approximated by

$$
H\left(t, f_{\mathrm{c}}\right)=\sqrt{\frac{\sigma_{0}^{2}}{N}} \sum_{k=1}^{N} \exp \left(\mathrm{j} \theta_{k}\right) \exp \left(-\mathrm{j} 2 \pi f_{\mathrm{c}} \tau_{k}(t)\right),
$$

as $N \rightarrow \infty$, where $\sigma_{0}^{2}$ is the total channel power. $\theta_{k}$ is the phase and $\tau_{k}(t)$ is the time-variant delay of the scatterer $k$ in (16) . The correlation function for the time-variant transfer function is according to [4] defined as

$$
\begin{array}{r}
r_{M}\left(t, t+\Delta t, f_{\mathrm{c}}, f_{\mathrm{c}}+\Delta f\right)= \\
=\mathbb{E}\left\{H^{*}\left(f_{\mathrm{c}}, t\right) H\left(t, t+\Delta t, f_{\mathrm{c}}+\Delta f\right)\right\},
\end{array}
$$

where $\mathbb{E}\{\cdot\}$ is an expectation operator, which is applied over the ensemble. After inserting (16) into (17) and simplifying the result, we obtain the following

$$
\begin{aligned}
& r_{M}\left(t, t+\Delta t, f_{\mathrm{c}}, f_{\mathrm{c}}+\Delta f\right)= \\
& =\frac{\sigma_{0}^{2}}{N} \mathbb{E}\left\{\sum_{\substack{k=1 \\
k}}^{N} \sum_{\substack{l=1 \\
l \neq k}}^{N} \mathrm{e}^{\mathrm{j} \theta_{l}-\mathrm{j} \theta_{k}} \mathrm{e}^{\mathrm{j} 2 \pi\left(f_{\mathrm{c}} \tau_{k}(t)-\left(f_{\mathrm{c}}+\Delta f\right) \tau_{l}(t+\Delta t)\right)}\right. \\
& \left.+\sum_{k=1}^{N} \mathrm{e}^{\mathrm{j} 2 \pi\left(f_{\mathrm{c}}\left(\tau_{k}(t)-\tau_{k}(t+\Delta t)\right)\right)} \mathrm{e}^{-\mathrm{j} 2 \pi \Delta f \tau_{k}(t+\Delta t)}\right\}
\end{aligned}
$$

We define the Doppler frequency as negative derivative of the delay

$$
f_{\mathrm{c}} \frac{\left(\tau_{k}(t)-\tau_{k}(t+\Delta t)\right)}{\Delta t} \Delta t \approx-f_{\mathrm{c}} \frac{\mathrm{d} \tau(t)}{\mathrm{d} t} \Delta t=f_{\mathrm{d}}(t) \Delta t .
$$

This approximation becomes exact when $\Delta t \rightarrow 0$, or if $f_{\mathrm{d}}(t)$ is a constant function.

Invoking the US assumption, it can be verified that the terms in the double sum in (18) become zero. Additionally, by using expression (66) in [4], we can similarly obtain

$$
\begin{aligned}
& r_{M}(t, t+\Delta t, \Delta f) \approx \\
& \approx \frac{\sigma_{0}^{2}}{N} \mathbb{E}\left\{\sum_{k=1}^{N} \exp \left(\mathrm{j} 2 \pi f_{\mathrm{d}, k}(t) \Delta t\right) \exp \left(-\mathrm{j} 2 \pi \Delta f \tau_{k}(t)\right)\right\} .
\end{aligned}
$$

Since ensemble average and summation are exchangeable because of linearity, and due to the identical distribution of the terms under the summation operator in (20), the correlation function becomes

$$
r_{M}(t, t+\Delta t, \Delta f) \approx \sigma_{0}^{2} \mathbb{E}\left\{\mathrm{e}^{\mathrm{j} 2 \pi\left(f_{\mathrm{d}}(t) \Delta t-\Delta f \tau(t)\right)}\right\}
$$

for some $f_{\mathrm{d}}(t)$ and $\tau(t)$. Note that due to the invalidity of the WSS assumption the resulting expression is time-variant, but can be calculated point-wise in time for any $t=t^{*}$ by the ensemble average. For a fixed time $t^{*}$ the timevariant stochastic processes $f_{\mathrm{d}}(t)$ and $\tau(t)$ become stochastic variables $f_{\mathrm{d}}:=f_{\mathrm{d}}\left(t^{*}\right)$ and $\tau:=\xi \tau_{\text {los }}\left(t^{*}\right)$ according to [21]. Thus by evaluating (21) we obtain

$$
\begin{aligned}
r_{M}\left(t^{*}, t^{*}+\Delta t, \Delta f\right) \approx & \sigma_{0}^{2} \int_{-\infty}^{\infty} \int_{-\infty}^{\infty} p\left(t^{*} ; f_{\mathrm{d}}, \xi\right) \\
& \exp \left(\mathrm{j} 2 \pi\left(f_{\mathrm{d}} \Delta t-\Delta f \xi \tau_{\mathrm{los}}\right)\right) \mathrm{d} \xi \mathrm{d} f_{\mathrm{d}} .
\end{aligned}
$$

In the analysis of time-variant channels performed by Bello in his seminal paper [4, Section IV. C.] the same correlation function - up to a constant factor - can be obtained by double Fourier transform of the time-variant power spectral density 


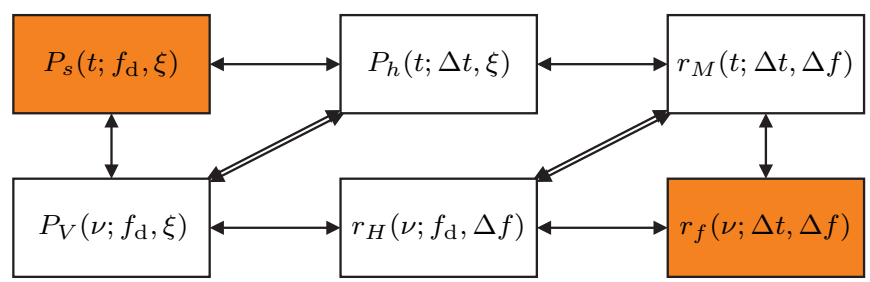

Fig. 2. Relationships for channel correlation functions for a US channel according to [4] with arrows marking Fourier transforms. The two shaded blocks are extensions to Fig. 8 in [4]. The variables $\Delta t$ and $\Delta f$ are defined as relative shifts with respect to $t$ and $f_{\mathrm{c}}$, respectively.

$P_{s}\left(t ; f_{\mathrm{d}}, \xi\right)$ for the US case, which is also known as timevariant scattering function. The correlation becomes

$$
\begin{array}{r}
r_{M}\left(t^{*}, t^{*}+\Delta t, \Delta f\right)=\int_{-\infty}^{\infty} P_{h}\left(t^{*} ; \Delta t, \xi\right) \mathrm{e}^{-\mathrm{j} 2 \pi \Delta f \xi \tau_{\text {los }}} \mathrm{d} \xi \\
=\int_{-\infty}^{\infty} \int_{-\infty}^{\infty} P_{s}\left(t^{*} ; f_{\mathrm{d}}, \xi\right) \mathrm{e}^{\mathrm{j} 2 \pi\left(f_{\mathrm{d}} \Delta t-\Delta f \xi \tau_{\text {los }}\right)} \mathrm{d} \xi \mathrm{d} f_{\mathrm{d}},
\end{array}
$$

where $P_{h}\left(t^{*} ; \Delta t, \xi\right)$ is the delay-spread autocorrelation function according to [4]. The relationship between the different functions for the US case is shown in Fig. 2. In the limit, as $\Delta t \rightarrow 0,(22)$ becomes an exact equality. By comparing (22) and (23), we can argue that point-wise, i.e., for each $t=t^{*}$ and $\Delta t \rightarrow 0$, it follows that

$$
P_{s}\left(t^{*} ; f_{\mathrm{d}}, \xi\right) \propto p\left(t^{*} ; f_{\mathrm{d}}, \xi\right) .
$$

Observe, that the exact proportionality is only given for one point in time $t=t^{*}$, which reflects the absence of the WSS property. Yet, from a practical perspective, the pdf $p\left(t^{*} ; f_{\mathrm{d}}, \xi\right)$ is still a good approximation in the stationarity region of the channel, when (19) holds.

In a similar fashion, using the properties of the Fourier transform, it can be shown that

$$
\begin{aligned}
& \int_{-\infty}^{\infty} P_{h}\left(t^{*} ; \Delta t, \xi\right) \mathrm{e}^{-\mathrm{j} 2 \pi \Delta f \xi \tau_{\text {los }}} \mathrm{d} \xi \\
& \quad \approx \sigma_{0}^{2} \int_{-\infty}^{\infty} \int_{-\infty}^{\infty} p\left(t^{*} ; f_{\mathrm{d}}, \xi\right) \mathrm{e}^{\mathrm{j} 2 \pi\left(f_{\mathrm{d}} \Delta t-\Delta f \xi \tau_{\text {los }}\right)} \mathrm{d} \xi \mathrm{d} f_{\mathrm{d}} \\
& \approx \sigma_{0}^{2} \int_{-\infty}^{\infty} p\left(t^{*} ; \xi\right) \Phi\left(t^{*} ; \Delta t \mid \xi\right) \mathrm{e}^{-\mathrm{j} 2 \pi \Delta f \xi \tau_{\text {los }}} \mathrm{d} \xi
\end{aligned}
$$

where we used the fact that $p\left(t^{*} ; f_{\mathrm{d}}, \xi\right)=p\left(t^{*} ; \xi\right) p\left(t^{*} ; f_{\mathrm{d}} \mid \xi\right)$ and $\Phi\left(t^{*} ; \Delta t \mid \xi\right)$ is the characteristic function of $p\left(t^{*} ; f_{\mathrm{d}} \mid \xi\right)$ in (11). With $\Delta t \rightarrow 0$, the approximation in (25) becomes exact. For a fixed delay $\xi=\xi^{*}$ or if $p\left(t^{*} ; \xi\right)$ is a constant function, we can state that the correlation function is proportional to the characteristic function of the Doppler pdf, i.e.,

$$
P_{h}\left(t^{*} ; \Delta t, \xi^{*}\right) \propto \Phi\left(t^{*} ; \Delta t \mid \xi^{*}\right) .
$$

\section{Analysis of the Vehicular Channel CHARACTERISTIC FUNCTION AND DOPPLER PDF}

In this section, we present an application of the analysis presented in the last section to general 2D vehicular scenarios, in which both vehicles drive along arbitrary velocity vectors $\mathbf{v}_{\mathrm{t}}$ and $\mathbf{v}_{\mathrm{r}}$. Please note that the velocity vectors $\mathbf{v}_{\mathrm{t}}$ and $\mathbf{v}_{\mathrm{r}}$ and all Doppler frequencies are typically functions of time $t$; we will drop the explicit time dependency for these parameters to simplify the results.

In particular, we derive and analyze the closed-form expressions for Doppler pdf, characteristic function, mean Doppler, and Doppler spread obtained for two cases: $\xi=1$ and $\xi \rightarrow \infty$. Furthermore, the limiting frequencies for the Doppler pdf are determined for $\xi=1+\epsilon$ and $\xi \rightarrow \infty$, where $\xi=1$ means the LOS delay, $\xi=1+\epsilon$ is an $\epsilon$-vicinity of the LOS delay, and $\xi=\infty$ is an infinite delay, respectively. The different cases allow to compare the newly obtained results to those already known from the literature.

\section{A. Line-of-Sight Delay $(\xi=1)$}

For the LOS case, the expressions in (11), (12) and (13) can be simplified. Thus, we obtain the following closed-form solutions

$$
\begin{aligned}
& \lim _{\xi \rightarrow 1} \Phi(t ; u \mid \xi)=\exp \left(\mathrm{j} 2 \pi u \frac{\left(v_{\mathrm{t}}^{z}-v_{\mathrm{r}}^{z}\right)}{c} f_{\mathrm{c}}\right), \\
& \lim _{\xi \rightarrow 1} p\left(t ; f_{\mathrm{d}} \mid \xi\right)=\delta\left(f_{\mathrm{d}}-f_{\text {los }}\right), \\
& \lim _{\xi \rightarrow 1} \mu(t ; \xi)=\frac{\left(v_{\mathrm{t}}^{z}-v_{\mathrm{r}}^{z}\right)}{c} f_{\mathrm{c}}=f_{\text {los }}, \lim _{\xi \rightarrow 1} \sigma(t ; \xi)=0 .
\end{aligned}
$$

Observe that the characteristic function for the LOS path is a complex exponential function $\lim _{\xi \rightarrow 1} \Phi(t ; u \mid \xi)=$ $\exp \left\{\mathrm{j} 2 \pi u f_{\text {los }}\right\}$, which is an expected result. As a consequence, the Doppler spread $\lim _{\xi \rightarrow 1} \sigma(t ; \xi)=0$ is zero for the LOS with the mean Doppler being equal to the LOS Doppler frequency $\lim _{\xi \rightarrow 1} \mu(t ; \xi)=f_{\text {los }}$. In other words, the Doppler spectrum is a Dirac distribution at the LOS Doppler frequency.

\section{B. Behavior of Doppler Spectrum Close to LOS $(\xi=1+\epsilon)$}

An analysis of the Doppler pdf has revealed that the latter exhibits a very abrupt transition from $\xi=1$, i.e., from LOS, to $\xi>1$. Specifically, in the $\epsilon$-vicinity of the LOS, the Doppler frequency abruptly increases from a Dirac impulse for $\xi=1$ to a non-zero Doppler spectral width. Such a behavior can be explained as follows. In the case $\xi=1$, the ellipse on which scatterers are located is a line. Yet, for $\xi=1+\epsilon$ the line opens into an ellipse with very large eccentricity $\frac{1}{\xi}$. As a consequence, in the vicinity of the LOS, the spectral width abruptly grows from zero width to a bandwidth larger than zero. By studying the Doppler pdf for different velocity vectors of transmitter and receiver in the $\xi=1+\epsilon$ region, we determined that the support of the Doppler spectrum has three regions in this case characterized by the limiting frequencies 


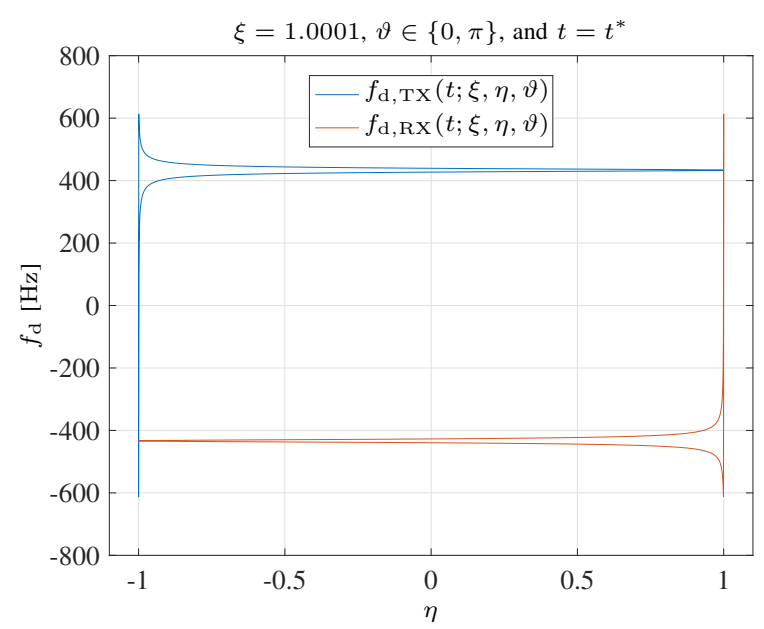

Fig. 3. Behavior of Doppler frequency close to the LOS in dependence on $\eta$ with $\mathbf{v}_{\mathrm{t}}=[90,0,90]^{\mathrm{T}} \mathrm{km} / \mathrm{h}$ and $\mathbf{v}_{\mathrm{r}}=[90,0,90]^{\mathrm{T}} \mathrm{km} / \mathrm{h}$.

$f_{\mathrm{b} 1}, f_{\mathrm{b} 2}, f_{\mathrm{b} 3}$, and $f_{\mathrm{b} 4}$. These are given by the following expressions

$$
\begin{aligned}
& f_{\mathrm{b} 1,2}(t)=\frac{\left( \pm\left\|\mathbf{v}_{\mathrm{t}}\right\|-v_{\mathrm{r}}^{z}\right)}{c} f_{\mathrm{c}} \\
& f_{\mathrm{b} 3,4}(t)=\frac{\left( \pm\left\|\mathbf{v}_{\mathrm{r}}\right\|+v_{\mathrm{t}}^{z}\right)}{c} f_{\mathrm{c}} .
\end{aligned}
$$

These expressions can also be used to determine the maximum and minimum Doppler frequency in the general case in the $\epsilon$ vicinity of the LOS. In the following, we show how these limiting frequencies can be computed.

First, from (7) we can see that the Doppler frequency possesses two singularities at points $s_{1}:=(\xi=1, \eta=-1)$ and $s_{2}:=(\xi=1, \eta=1)$; these are the coordinates of the TX and the RX, respectively.

Let us consider (7) in the vicinity of $s_{1}$. Thus, in the V2V case, i.e., $\vartheta \in\{0, \pi\}$, in the vicinity of $s_{1}$ the Doppler frequency $f_{\mathrm{d}}(t ; \xi, \eta, \vartheta) \equiv f_{\mathrm{d}}^{\left[s_{1}\right]}(t ; \xi, \eta)$ can be represented as the corresponding TX and RX contributions $f_{\mathrm{d}}^{\left[s_{1}\right]}(t ; \xi, \eta)=$ $f_{\mathrm{d}, \mathrm{TX}}^{\left[s_{1}\right]}(t ; \xi, \eta)+f_{\mathrm{d}, \mathrm{RX}}^{\left[s_{1}\right]}(t ; \xi, \eta)$. Subsequently, we study the extrema of $f_{\mathrm{d}}^{\left[s_{1}\right]}(t ; \xi, \eta)$ as a function of $\eta$. Unfortunately, the straightforward analysis of $f_{\mathrm{d}}^{\left[s_{1}\right]}(t ; \xi, \eta)$ becomes analytically intractable since the derivative of $f_{\mathrm{d}}^{\left[s_{1}\right]}(t ; \xi, \eta)$ with respect to $\eta$ is a 6 th order polynomial. Instead, we use the fact that the term $f_{\mathrm{d}, \mathrm{RX}}^{\left[s_{1}\right]}(t ; \xi, \eta)$ becomes almost independent of $\eta$ in the vicinity of $s_{1}$, which can be seen Fig. 3. Thus, by setting $\xi=1$ it can be approximated as follows

$$
f_{\mathrm{d}, \mathrm{RX}}^{\left[s_{1}\right]}(t ; \xi, \eta) \approx \lim _{\eta \rightarrow-1} f_{\mathrm{d}, \mathrm{RX}}^{\left[s_{1}\right]}(t ; \xi=1, \eta)=-\frac{v_{\mathrm{r}}^{z}}{c} f_{\mathrm{c}} .
$$

This result corresponds to the receiver contribution to $f_{\text {los }}$ in (27). Therefore, the receiver contribution is only a constant offset and does not influence the location of the extrema. Inserting (30) back into $f_{\mathrm{d}}^{\left[s_{1}\right]}(t ; \xi, \eta)$, we can approximate it using the following expression

$$
\begin{aligned}
& f_{\mathrm{d}}^{\left[s_{1}\right]}(t ; \xi, \eta) \approx \\
& \quad \approx\left( \pm \frac{\sqrt{\left(\xi^{2}-1\right)\left(1-\eta^{2}\right)}}{\xi+\eta} v_{\mathrm{t}}^{x}+\frac{\xi \eta+1}{\xi+\eta} v_{\mathrm{t}}^{z}-v_{\mathrm{r}}^{z}\right) \frac{f_{\mathrm{c}}}{c} .
\end{aligned}
$$

Now, we take the derivative of $f_{\mathrm{d}}^{\left[s_{1}\right]}(t ; \xi, \eta)$ in (31) with respect to $\eta$ and set it to zero. The solution is found at $\eta_{0, \mathrm{TX}}$ given by (32). Inserting (32) into (31) and simplifying the resulting expression finally leads to two extremal frequencies

$$
f_{\mathrm{b} 1,2}(t)=\frac{\left( \pm\left\|\mathbf{v}_{\mathrm{t}}\right\|-v_{\mathrm{r}}^{z}\right)}{c} f_{\mathrm{c}}
$$

which match the result in (28).

For the second point $s_{2}$, a similar computation can be performed. In the vicinity of $s_{2}$ the Doppler frequency $f_{\mathrm{d}}^{\left[s_{2}\right]}(t ; \xi, \eta)$ can be likewise decomposed as $f_{\mathrm{d}}^{\left[s_{2}\right]}(t ; \xi, \eta)=$ $f_{\mathrm{d}, \mathrm{TX}}^{\left[s_{2}\right]}(t ; \xi, \eta)+f_{\mathrm{d}, \mathrm{RX}}^{\left[s_{2}\right]}(t ; \xi, \eta)$. As in the previous case, we simplify the expression for the Doppler frequency close to $s_{2}$ by using the fact that $f_{\mathrm{d}, \mathrm{TX}}^{\left[s_{2}\right]}(t ; \xi, \eta)$ becomes almost independent of $\eta$ in the vicinity of $s_{2}$ as shown in Fig. 3. Thus, by setting $\xi=1$ it can be approximated as follows

$$
f_{\mathrm{d}, \mathrm{TX}}^{\left[s_{2}\right]}(t ; \xi, \eta) \approx \lim _{\eta \rightarrow 1} f_{\mathrm{d}, \mathrm{TX}}^{\left[s_{2}\right]}(t ; \xi=1, \eta)=\frac{v_{\mathrm{t}}^{z}}{c} f_{\mathrm{c}} .
$$

Inserting (34) into $f_{\mathrm{d}}^{\left[s_{2}\right]}(t ; \xi, \eta)$, we can approximate it using the following expression

$$
\begin{aligned}
& f_{\mathrm{d}}^{\left[s_{2}\right]}(t ; \xi, \eta) \approx \\
& \quad \approx\left( \pm \frac{\sqrt{\left(\xi^{2}-1\right)\left(1-\eta^{2}\right)}}{\xi-\eta} v_{\mathrm{r}}^{x}+\frac{\xi \eta-1}{\xi-\eta} v_{\mathrm{r}}^{z}+v_{\mathrm{t}}^{z}\right) \frac{f_{\mathrm{c}}}{c} .
\end{aligned}
$$

By setting the derivative of the latter expression with respect to $\eta$ to zero, we find its extrema at $\eta_{0, \mathrm{Rx}}$ given by (36). Similar to the first case, we compute that close to $s_{2}$ the Doppler frequency will have two extrema given by

$$
f_{\mathrm{b} 3,4}(t)=\frac{\left( \pm\left\|\mathbf{v}_{\mathrm{r}}\right\|+v_{\mathrm{t}}^{z}\right)}{c} f_{\mathrm{c}}
$$

This result concurs with (29).

$$
\begin{aligned}
& \eta_{0, \mathrm{TX}}=\frac{ \pm \sqrt{\left(v_{\mathrm{t}}^{x}\right)^{2}\left(v_{\mathrm{t}}^{z}\right)^{2} \xi^{4}-2\left(v_{\mathrm{t}}^{x}\right)^{2}\left(v_{\mathrm{t}}^{z}\right)^{2} \xi^{2}+\left(v_{\mathrm{t}}^{x}\right)^{2}\left(v_{\mathrm{t}}^{z}\right)^{2}+\left(v_{\mathrm{t}}^{z}\right)^{4} \xi^{4}-2\left(v_{\mathrm{t}}^{z}\right)^{4} \xi^{2}+\left(v_{\mathrm{t}}^{z}\right)^{4}}-\left(v_{\mathrm{t}}^{x}\right)^{2} \xi}{\left(v_{\mathrm{t}}^{x}\right)^{2} \xi^{2}+\left(v_{\mathrm{t}}^{z}\right)^{2} \xi^{2}-\left(v_{\mathrm{t}}^{z}\right)^{2}} . \\
& \eta_{0, \mathrm{RX}}=\frac{ \pm \sqrt{\left(v_{\mathrm{r}}^{x}\right)^{2}\left(v_{\mathrm{r}}^{z}\right)^{2} \xi^{4}-2\left(v_{\mathrm{r}}^{x}\right)^{2}\left(v_{\mathrm{r}}^{z}\right)^{2} \xi^{2}+\left(v_{\mathrm{r}}^{x}\right)^{2}\left(v_{\mathrm{r}}^{z}\right)^{2}+\left(v_{\mathrm{r}}^{z}\right)^{4} \xi^{4}-2\left(v_{\mathrm{r}}^{z}\right)^{4} \xi^{2}+\left(v_{\mathrm{r}}^{z}\right)^{4}}+\left(v_{\mathrm{r}}^{x}\right)^{2} \xi}{\left(v_{\mathrm{r}}^{x}\right)^{2} \xi^{2}+\left(v_{\mathrm{r}}^{z}\right)^{2} \xi^{2}-\left(v_{\mathrm{r}}^{z}\right)^{2}} .
\end{aligned}
$$




\section{Infinite Delay $(\xi \rightarrow \infty)$}

For the infinite delay case, we obtain the following closedform solutions

$$
\begin{aligned}
& \lim _{\xi \rightarrow \infty} \Phi(t ; u \mid \xi)=J_{0}\left(2 \pi u \frac{\left\|\mathbf{v}_{\mathrm{t}}+\mathbf{v}_{\mathbf{r}}\right\|}{c} f_{\mathrm{c}}\right), \\
& \lim _{\xi \rightarrow \infty} p\left(t ; f_{\mathrm{d}} \mid \xi\right)=\frac{1}{\pi\left|f_{\mathrm{e} 1,2}\right| \sqrt{1-\left(\frac{f_{\mathrm{d}}}{f_{\mathrm{e} 1,2}}\right)^{2}}}, \\
& \lim _{\xi \rightarrow \infty} \mu(t ; \xi)=0, \lim _{\xi \rightarrow \infty} \sigma(t ; \xi)=\frac{\left(\left\|\mathbf{v}_{\mathrm{t}}+\mathbf{v}_{\mathrm{r}}\right\|\right)}{\sqrt{2} c} f_{\mathrm{c}}, \\
& f_{\mathrm{e} 1,2}(t)= \pm \frac{\left(\left\|\mathbf{v}_{\mathrm{t}}+\mathbf{v}_{\mathrm{r}}\right\|\right)}{c} f_{\mathrm{c}},
\end{aligned}
$$

where $f_{\mathrm{e} 1,2}(t)$ are the limiting frequencies of the Doppler pdf. The characteristic function $\Phi(t ; u \mid \xi)$ becomes a zerothorder Bessel function with the Doppler pdf following the well-known Jakes spectrum. Both results are well-known from classical theory, but it is shown here that the result is valid for arbitrary velocity vectors of transmitter and receiver; as such the computed expressions generalize well the known relationships between Doppler spectrum and the corresponding correlation function. Naturally, in this case the mean Doppler becomes zero, i.e., $\lim _{\xi \rightarrow \infty} \mu(t ; \xi)=0$ due to the symmetry of the Doppler pdf. The Doppler spread is constant and it can be computed using the limiting frequencies of the Doppler pdf $\left|f_{\mathrm{e} 1,2}\right|$ scaled by $\frac{1}{\sqrt{2}}$, i.e., $\lim _{\xi \rightarrow \infty} \sigma(t ; \xi)=\frac{\left|f_{\mathrm{e} 1,2}\right|}{\sqrt{2}}$ using a known expression from the Jakes spectrum.

The proof for the limiting frequencies for $\xi \rightarrow \infty$ is obtained as follows. Again, for the V2V case, i.e., $\vartheta \in\{0, \pi\}$ the Doppler frequency in (7) becomes

$$
\lim _{\xi \rightarrow \infty} f_{\mathrm{d}}(t ; \xi, \eta)=\left( \pm \sqrt{1-\eta^{2}}\left(v_{\mathrm{t}}^{x}+v_{\mathrm{r}}^{x}\right)+\eta\left(v_{\mathrm{t}}^{z}+v_{\mathrm{r}}^{z}\right)\right) \frac{f_{\mathrm{c}}}{c} .
$$

As before the extrema are determined by setting the derivative of (39) to zero. The solution is obtained for

$$
\eta_{0}= \pm \frac{\left(v_{\mathrm{t}}^{z}+v_{\mathrm{r}}^{z}\right)}{\sqrt{\left(v_{\mathrm{t}}^{x}+v_{\mathrm{r}}^{x}\right)^{2}+\left(v_{\mathrm{t}}^{z}+v_{\mathrm{r}}^{z}\right)^{2}}} .
$$

The expression for the limiting frequencies is obtained by inserting (40) into (39) and simplifying the result, which leads to

$$
f_{\mathrm{e} 1,2}(t)= \pm \frac{\left(\left\|\mathbf{v}_{\mathrm{t}}+\mathbf{v}_{\mathrm{r}}\right\|\right)}{c} f_{\mathrm{c}} .
$$

Note that the limiting frequencies are dependent on the vector sum of the velocity vectors of transmitter and receiver as given in (41).

\section{REsults}

Let us consider several special cases which follow from (27)-(29) and (38) and investigate some typical vehicular scenarios. These scenarios are summarized in Table I. The evaluation is done at an arbitrary, but fixed time $t=t^{*}$ for every scenario. These scenarios readily occur on highways or crossings, with the exception of Scenario 2. A small lateral distance would have to be included to make Scenario 2 more realistic; this scenario is presented to complement Scenario 1.
Note that the characteristic function, Doppler pdf, mean Doppler, and Doppler spread cannot be computed in closedform for the cases $1<\xi<\infty$. Nonetheless, a numerical evaluation can be computed, which is presented in the following.

Let us study the obtained expressions in more detail. Thus, we fix some of the channel parameters to realistic values and numerically generate the characteristic function, the Doppler pdf, as well as the mean Doppler and Doppler spread for the scenarios summarized in Table I. We set the carrier frequency to $f_{\mathrm{c}}=5.2 \mathrm{GHz}$, which is close to the intended $\mathrm{V} 2 \mathrm{~V}$ frequency in order to make the results comparable to those in the literature. Note that the results are computed for the carrier frequency only. In general, the equations are also valid for wideband signals, since they can be represented as a sum of continuous waves. However, the limited bandwidth and time observability means that the results in the Doppler delay domain would be convolved with a sinc-function in both delay and Doppler frequency and thus would spread the energy of the Dirac measure in the delay Doppler domain, see [36].

In order to visualize the case close to the line-of-sight, i.e., $\xi=1+\epsilon$, we approximate it by $\xi=1.01$ and for infinite delay, i.e., $\xi \rightarrow \infty$, we set $\xi=1000$ which is sufficiently large to represent the effect of an infinite scatterer delay. Let us stress again that the results are general and valid for any delay, since $\xi$ specifies the relative delay that is normalized by the LOS delay. The corresponding absolute delay is obtained by $\tau\left(t^{*}\right)=\frac{2 l \xi}{c}$. Furthermore, it is assumed that for a pdf $p\left(t^{*} ; \xi, f_{\mathrm{d}}\right)=p\left(t^{*} ; f_{\mathrm{d}} \mid \xi\right) p\left(t^{*} ; \xi\right)$ the marginal $p\left(t^{*} ; \xi\right)$ is constant. This implies that the "cuts" through $p\left(t^{*} ; \xi, f_{\mathrm{d}}\right)$ along $\xi$ will effectively give a delay-dependent Doppler pdf up to a proportionality constant that will make $p\left(t^{*} ; \xi, f_{\mathrm{d}}\right)$ integrate to one. Note also that when the delaydependent Doppler pdf $p\left(t^{*} ; f_{\mathrm{d}} \mid \xi\right)$ is not symmetric, the delaydependent characteristic function naturally becomes complex, which follows from the properties of the Fourier transform.

Therefore, we show only the real part of the characteristic function, since this part corresponds to well-known correlation functions like the cosine or Bessel functions.

We begin with Scenario 1. The results are summarized in Fig. 4, Fig. 5, and Fig. 6. The LOS has a constant characteristic function, which means it is time-invariant, and the LOS frequency is at $f_{\text {los }}=0 \mathrm{~Hz}$. Since both vehicles drive in the same direction with the same speed, the result is reasonable. The limiting frequencies close to the LOS are $f_{\mathrm{b}} \in\{-866.67,0,866.67\} \mathrm{Hz}$ and thus a spectral width of $1733.33 \mathrm{~Hz}$ is obtained. Note that this means an abrupt transition of the spectral width as soon as $\xi$ grows to $1+\epsilon$ for small $\epsilon>0$. For $\xi=1.01$ the influence of the LOS in the pdf is obvious in Fig. 5 and the characteristic function becomes similar to a triangle. For $\xi=1.5$, the characteristic function already shows similarity to a Bessel function as can be seen in Fig. 4, but with a wider mainlobe. For $\xi \rightarrow \infty$, we obtain the Bessel function with limiting frequencies of $f_{\mathrm{e}} \in$ $\{-866.67,866.67\} \mathrm{Hz}$ with a spectral width of $1733.33 \mathrm{~Hz}$. The mean Doppler $\mu\left(t^{*} ; \xi\right)$ remains zero for all $\xi \in[1, \infty)$, since the Doppler pdf is symmetric, but the Doppler spread 
TABLE I

RESULTS FOR ELEMENTARY VEHICULAR SCENARIOS

\begin{tabular}{|c|c|c|c|c|c|c|}
\hline & & Scenario 1 & Scenario 2 & Scenario 3 & Scenario 4 & Scenario 5 \\
\hline $\begin{array}{l}\frac{3}{0} \\
\frac{6}{20} \\
\frac{1}{2}\end{array}$ & $\begin{array}{l}\mathbf{v}_{\mathrm{t}} \\
\mathrm{v}_{\mathrm{r}}\end{array}$ & $\begin{array}{c}{\left[0,0, v_{\mathrm{t}}^{z}\right]^{\mathrm{T}}} \\
\mathbf{v}_{\mathrm{r}}=\mathbf{v}_{\mathrm{t}}\end{array}$ & $\begin{array}{l}{\left[0,0, v_{\mathrm{t}}^{z}\right]^{\mathrm{T}}} \\
\mathbf{v}_{\mathrm{r}}=-\mathbf{v}_{\mathrm{t}}\end{array}$ & $\begin{array}{c}{\left[v_{\mathrm{t}}^{x}, 0,0\right]^{\mathrm{T}}} \\
\mathbf{v}_{\mathrm{r}}=\mathbf{v}_{\mathrm{t}}\end{array}$ & $\begin{array}{l}{\left[v_{\mathrm{t}}^{x}, 0,0\right]^{\mathrm{T}}} \\
\mathbf{v}_{\mathrm{r}}=-\mathbf{v}_{\mathrm{t}}\end{array}$ & $\begin{array}{l}{\left[v_{\mathrm{t}}^{x}, 0,0\right]^{\mathrm{T}}} \\
{\left[0,0, v_{\mathrm{r}}^{z}\right]^{\mathrm{T}}}\end{array}$ \\
\hline 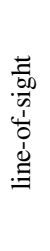 & $\begin{array}{l}\Phi\left(t^{*} ; u \mid \xi=1\right) \\
p\left(t^{*} ; f_{\mathrm{d}} \mid \xi=1\right) \\
\mu\left(t^{*} \mid \xi=1\right) \\
\sigma\left(t^{*} \mid \xi=1\right)\end{array}$ & $\begin{array}{c}1 \\
\delta\left(f_{\mathrm{d}}\right) \\
0 \\
0\end{array}$ & $\begin{array}{c}\exp \left(\mathrm{j} 2 \pi u \frac{2 v_{\mathrm{t}}^{z}}{c} f_{\mathrm{c}}\right) \\
\delta\left(f_{\mathrm{d}}-f_{\mathrm{los}}\right) \\
\frac{2 v_{\mathrm{t}}^{z}}{c} f_{\mathrm{c}} \\
0\end{array}$ & $\begin{array}{c}1 \\
\delta\left(f_{\mathrm{d}}\right) \\
0 \\
0\end{array}$ & $\begin{array}{c}1 \\
\delta\left(f_{\mathrm{d}}\right) \\
0 \\
0\end{array}$ & $\begin{array}{c}\exp \left(-\mathrm{j} 2 \pi u \frac{v_{\mathrm{r}}^{z}}{c} f_{\mathrm{c}}\right) \\
\delta\left(f_{\mathrm{d}}-f_{\mathrm{los}}\right) \\
-\frac{v_{\mathrm{r}}^{z}}{c} f_{\mathrm{c}} \\
0\end{array}$ \\
\hline $\begin{array}{l}20 \\
0 \\
0 \\
0 \\
0 \\
0 \\
0\end{array}$ & $\begin{array}{l}f_{\mathrm{b} 1,2}\left(t^{*}\right) \\
f_{\mathrm{b} 3,4}\left(t^{*}\right)\end{array}$ & $\begin{array}{c}0, \quad-\frac{2 v_{\mathrm{t}}^{z}}{c} f_{\mathrm{c}} \\
0, \quad \frac{2 v_{t}^{z}}{c} f_{\mathrm{c}}\end{array}$ & $\begin{array}{l}0, \quad \frac{2 v_{t}^{z}}{c} f_{\mathrm{c}} \\
0, \quad \frac{2 v_{t}^{z}}{c} f_{\mathrm{c}}\end{array}$ & $\begin{array}{l} \pm \frac{v_{\mathrm{t}}^{x}}{c} f_{\mathrm{c}} \\
\pm \frac{v_{\mathrm{t}}^{x}}{c} f_{\mathrm{c}}\end{array}$ & $\begin{array}{l} \pm \frac{v_{\mathrm{t}}^{x}}{c} f_{\mathrm{c}} \\
\pm \frac{v_{\mathrm{t}}^{x}}{c} f_{\mathrm{c}}\end{array}$ & $\begin{array}{c}\frac{ \pm v_{\mathrm{t}}^{x}-v_{\mathrm{r}}^{z}}{c} f_{\mathrm{c}} \\
\pm \frac{v_{\mathrm{r}}^{z}}{c} f_{\mathrm{c}}\end{array}$ \\
\hline 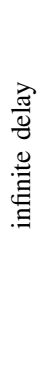 & $\begin{array}{l}\Phi\left(t^{*} ; u \mid \xi \rightarrow \infty\right) \\
p\left(t^{*} ; f_{\mathrm{d}} \mid \xi \rightarrow \infty\right) \\
\mu\left(t^{*} \mid \xi \rightarrow \infty\right) \\
\sigma\left(t^{*} \mid \xi \rightarrow \infty\right) \\
f_{\mathrm{e} 1,2}\left(t^{*}\right)\end{array}$ & $\begin{array}{c}J_{0}\left(2 \pi u \frac{2 v_{\mathrm{t}}^{z}}{c} f_{\mathrm{c}}\right) \\
\frac{1}{\pi\left|f_{\mathrm{e} 1,2}\right|} \sqrt{1-\left(\frac{f_{\mathrm{d}}}{f_{\mathrm{e} 1,2}}\right)^{2}} \\
0 \\
\frac{\sqrt{2} v_{\mathrm{t}}^{z}}{c} f_{\mathrm{c}} \\
\pm \frac{2 v_{\mathrm{t}}^{z}}{c} f_{\mathrm{c}}\end{array}$ & $\begin{array}{c}1 \\
\delta\left(f_{\mathrm{d}}\right) \\
0 \\
0\end{array}$ & $\begin{array}{c}J_{0}\left(2 \pi u \frac{2 v_{\mathrm{t}}^{x}}{c} f_{\mathrm{c}}\right) \\
\frac{1}{\pi\left|f_{\mathrm{e} 1,2}\right|} \sqrt{1-\left(\frac{f_{\mathrm{d}}}{f_{\mathrm{e} 1,2}}\right)^{2}} \\
0 \\
\frac{\sqrt{2} v_{\mathrm{t}}^{x}}{c} f_{\mathrm{c}} \\
\pm \frac{2 v_{\mathrm{t}}^{x}}{c} f_{\mathrm{c}}\end{array}$ & $\begin{array}{c}1 \\
\delta\left(f_{\mathrm{d}}\right)\end{array}$ & $\begin{array}{c}J_{0}\left(2 \pi u \frac{\sqrt{\left(v_{\mathrm{t}}^{x}\right)^{2}+\left(v_{\mathrm{r}}^{z}\right)^{2}}}{c} f_{\mathrm{c}}\right) \\
\frac{1}{\pi\left|f_{\mathrm{e} 1,2}\right| \sqrt{1-\left(\frac{f_{\mathrm{d}}}{f_{\mathrm{e} 1,2}}\right)^{2}}} \\
0 \\
\frac{\sqrt{\left(v_{\mathrm{t}}^{x}\right)^{2}+\left(v_{\mathrm{r}}^{z}\right)^{2}}}{\sqrt{2} c} f_{\mathrm{c}} \\
\pm \frac{\sqrt{\left(v_{\mathrm{t}}^{x}\right)^{2}+\left(v_{\mathrm{r}}^{z}\right)^{2}}}{c} f_{\mathrm{c}}\end{array}$ \\
\hline
\end{tabular}

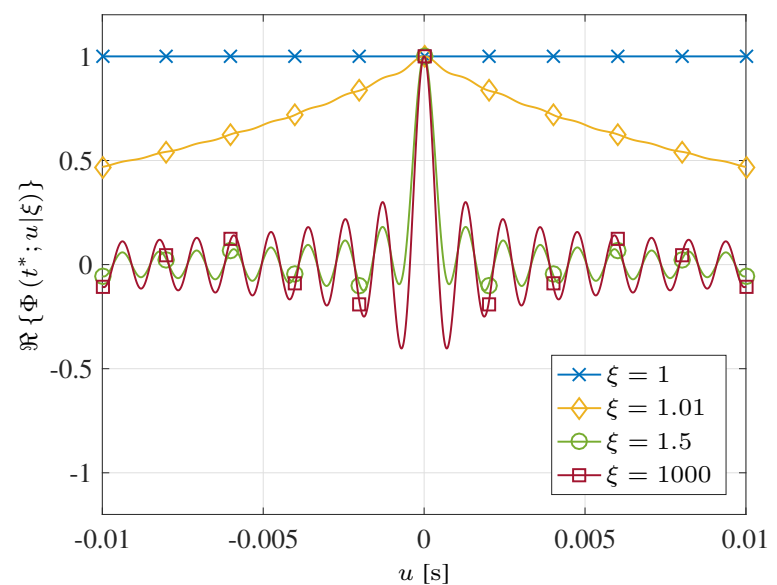

Fig. 4. Scenario 1: Real part of delay-dependent characteristic function $\Re\left\{\Phi\left(t^{*} ; u \mid \xi\right)\right\}$ for $\mathbf{v}_{\mathrm{t}}=[0,0,90]^{\mathrm{T}} \mathrm{km} / \mathrm{h}$ and $\mathbf{v}_{\mathrm{r}}=[0,0,90]^{\mathrm{T}} \mathrm{km} / \mathrm{h}$.

$\sigma\left(t^{*} ; \xi\right)$ is monotonically increasing to its calculated maximum value of $\lim _{\xi \rightarrow \infty} \sigma\left(t^{*} ; \xi\right)=612.83 \mathrm{~Hz}$, as shown in Fig. 6 .

Now, we consider Scenario 2. The results are presented in Fig. 7, Fig. 8, and Fig. 9. Here, the characteristic function of the LOS is a cosine function - the real part of a complex exponential - with a frequency of $f_{\text {los }}=866.67 \mathrm{~Hz}$, which means

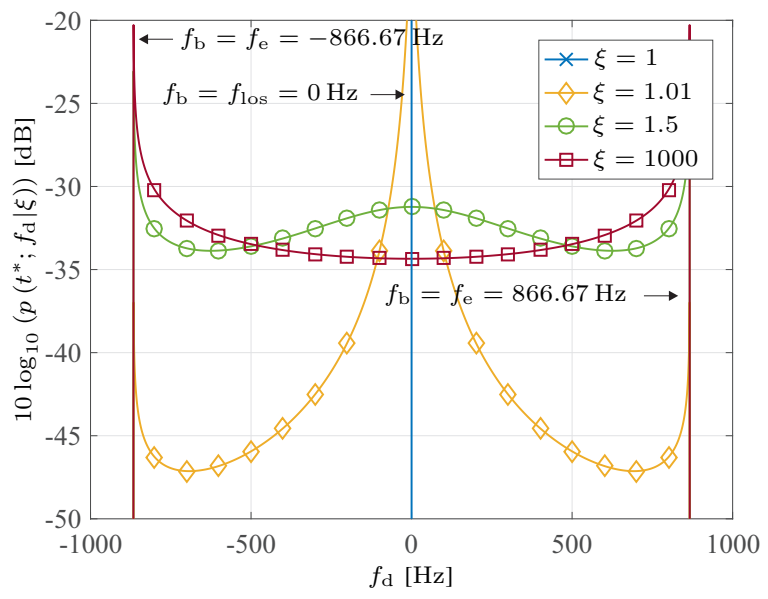

Fig. 5. Scenario 1: Delay-dependent Doppler pdf $p\left(t^{*} ; f_{\mathrm{d}} \mid \xi\right)$ and limiting frequencies $f_{\mathrm{b}}$ and $f_{\mathrm{e}}$ for $\mathbf{v}_{\mathrm{t}}=[0,0,90]^{\mathrm{T}} \mathrm{km} / \mathrm{h}$ and $\mathbf{v}_{\mathrm{r}}=[0,0,90]^{\mathrm{T}} \mathrm{km} / \mathrm{h}$.

the delay is decreasing between the vehicles. The limiting frequencies in the vicinity of the LOS are $f_{\mathrm{b}} \in\{0,866.67\} \mathrm{Hz}$ and thus a spectral width of $866.67 \mathrm{~Hz}$ is obtained. For $\xi=$ 1.01 , the limiting frequency of the pdf is slightly smaller as the LOS Doppler frequency in Fig. 8. The characteristic function resembles the product of a cosine and a triangle function in 


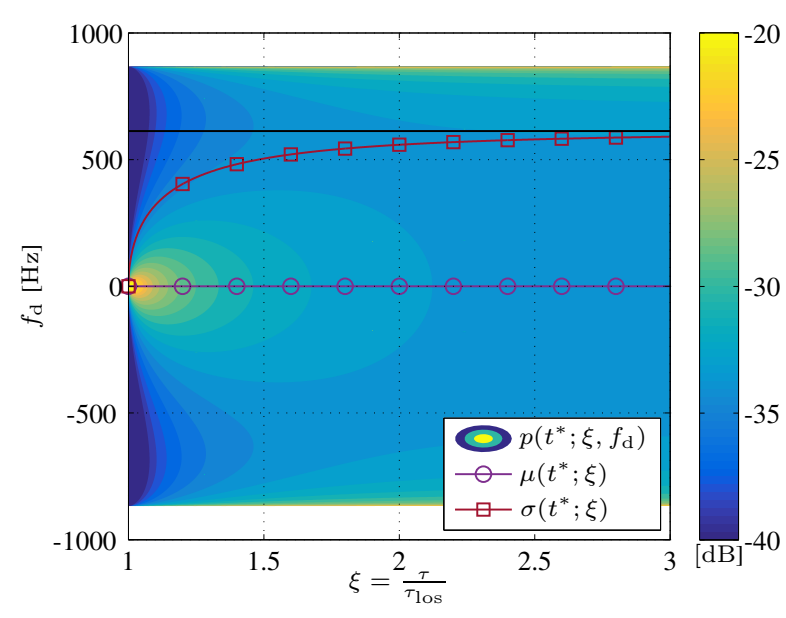

Fig. 6. Scenario 1: Joint delay Doppler pdf $p\left(t^{*} ; \xi, f_{\mathrm{d}}\right)$ with mean Doppler $\mu\left(t^{*} ; \xi\right)$, Doppler spread $\sigma\left(t^{*} ; \xi\right)$, and $\sigma$-asymptote for $\mathbf{v}_{\mathrm{t}}=$ $[0,0,90]^{\mathrm{T}} \mathrm{km} / \mathrm{h}$ and $\mathbf{v}_{\mathrm{r}}=[0,0,90]^{\mathrm{T}} \mathrm{km} / \mathrm{h}$.

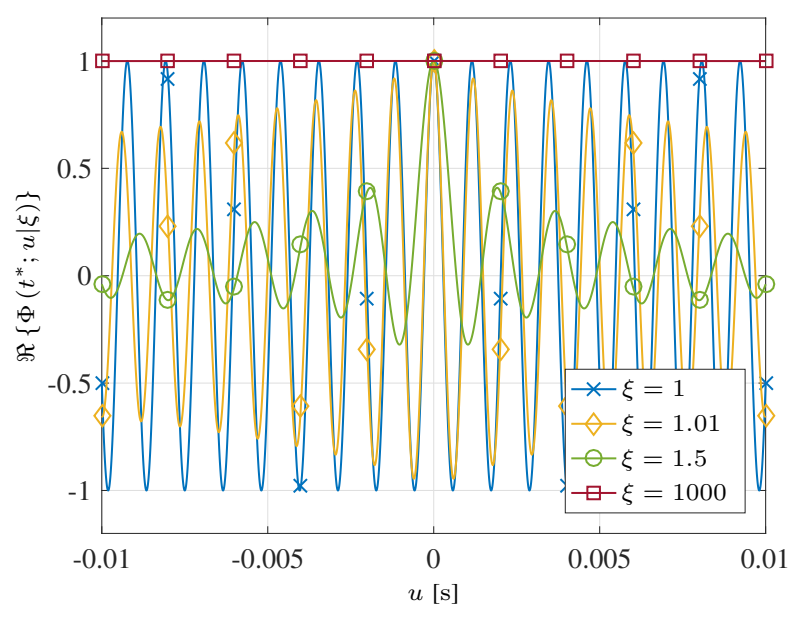

Fig. 7. Scenario 2: Real part of delay-dependent characteristic function $\Re\left\{\Phi\left(t^{*} ; u \mid \xi\right)\right\}$ for $\mathbf{v}_{\mathrm{t}}=[0,0,90]^{\mathrm{T}} \mathrm{km} / \mathrm{h}$ and $\mathbf{v}_{\mathrm{r}}=[0,0,-90]^{\mathrm{T}} \mathrm{km} / \mathrm{h}$.

Fig. 7. For $\xi=1.5$, the Doppler pdf becomes smaller and the attenuation of the cosine becomes more pronounced with the result approaching a Bessel function, as seen in Fig. 7. For $\xi \rightarrow \infty$, the characteristic function becomes 1 , so that there is no time-variance anymore. This is confirmed by the vanishing spectral width due to the limiting frequency of $f_{\mathrm{e}}=0 \mathrm{~Hz}$. Note that since the Doppler pdf has a support over positive frequencies, $\mu\left(t^{*} ; \xi\right)$ in Fig. 9 is strictly positive for all delays except $\xi \rightarrow \infty$, in which case it becomes zero. The Doppler spread $\sigma\left(t^{*} ; \xi\right)$ is non-zero for all frequencies with the exception of $\xi=1$ and $\xi \rightarrow \infty$.

The obtained results for Scenario 3 are presented in Fig. 10, Fig. 11, and Fig. 12. Here, the velocity vectors are orthogonal to the LOS line between the vehicles and the characteristic function is 1 in the LOS case. The limiting frequencies close to the LOS are $f_{\mathrm{b}} \in\{-433.33,433.33\} \mathrm{Hz}$ and thus a spectral width of $866.67 \mathrm{~Hz}$ is obtained. Here again there is the abrupt transition between $\xi=1$ and an $\epsilon$-environment of $\xi>1$ in the Doppler pdf. For $\xi=1.01$, the probability close to the LOS

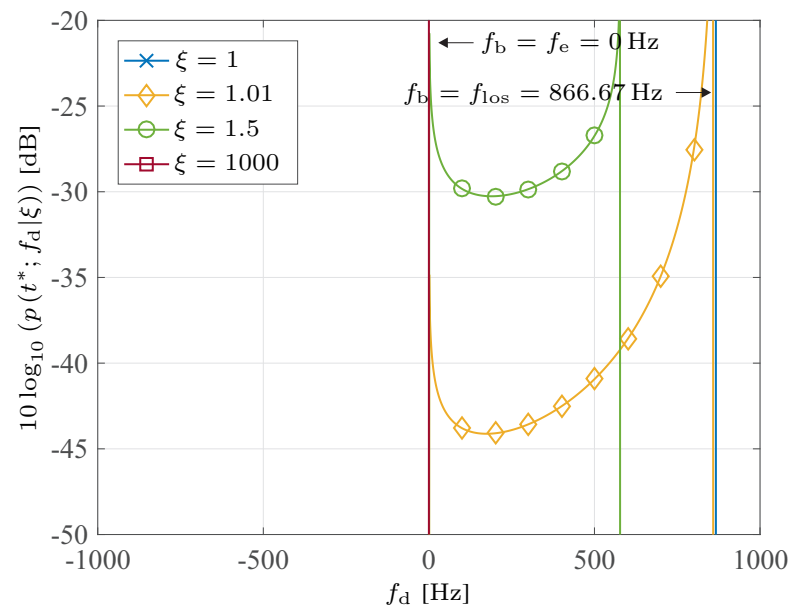

Fig. 8. Scenario 2: Delay-dependent Doppler pdf $p\left(t^{*} ; f_{\mathrm{d}} \mid \xi\right)$ and limiting frequencies $f_{\mathrm{b}}$ and $f_{\mathrm{e}}$ for $\mathbf{v}_{\mathrm{t}}=[0,0,90]^{\mathrm{T}} \mathrm{km} / \mathrm{h}$ and $\mathbf{v}_{\mathrm{r}}=$ $[0,0,-90]^{\mathrm{T}} \mathrm{km} / \mathrm{h}$.

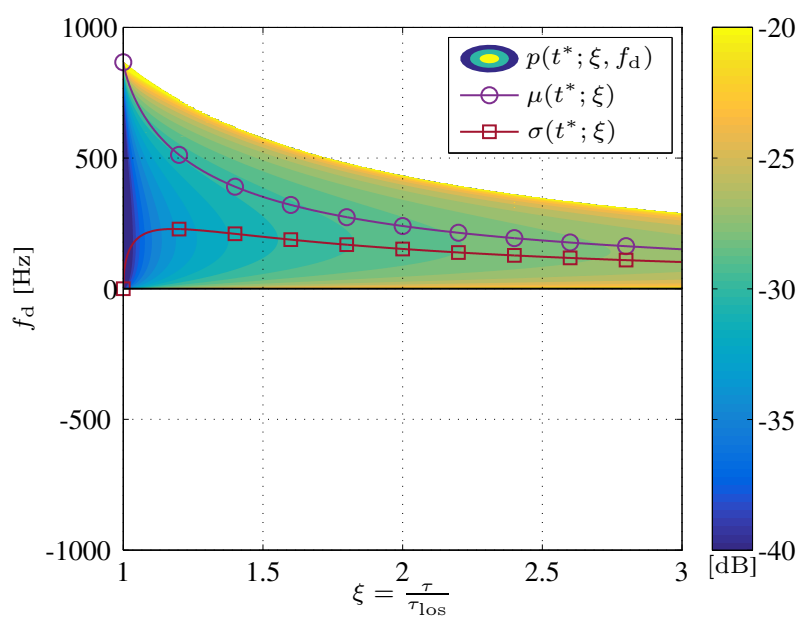

Fig. 9. Scenario 2: Joint delay Doppler pdf $p\left(t^{*} ; \xi, f_{\mathrm{d}}\right)$ with mean Doppler $\mu\left(t^{*} ; \xi\right)$, Doppler spread $\sigma\left(t^{*} ; \xi\right)$, and $\sigma$-asymptote for $\mathbf{v}_{\mathrm{t}}=$ $[0,0,90]^{\mathrm{T}} \mathrm{km} / \mathrm{h}$ and $\mathbf{v}_{\mathrm{r}}=[0,0,-90]^{\mathrm{T}} \mathrm{km} / \mathrm{h}$.

drops and separates the pdf in two halves in Fig. 11. The width of the characteristic function is very large as can be observed in Fig. 10. For $\xi=1.5$, the pdf becomes wider and the two outer spectra disappear. The corresponding characteristic function resembles the Bessel function. For $\xi \rightarrow \infty$, we obtain an exact Bessel function with limiting frequencies of $f_{\mathrm{e}} \in\{-866.67,866.67\} \mathrm{Hz}$ and the Doppler pdf reaches its maximum width of $1733.33 \mathrm{~Hz}$. The mean Doppler $\mu\left(t^{*} ; \xi\right)$ remains zero for all values of $\xi$ due to the symmetry of the Doppler pdf. The Doppler spread $\sigma\left(t^{*} ; \xi\right)$ however is monotonically increasing to its calculated maximum value of $\lim _{\xi \rightarrow \infty} \sigma\left(t^{*} ; \xi\right)=612.83 \mathrm{~Hz}$ in Fig. 12 .

The results for Scenario 4 are summarized in Fig. 13, Fig. 14, and Fig. 15. Here, time-variance is encountered for the LOS delay, since both vehicles drive in opposite directions with velocity vectors orthogonal to the major axis. The limiting frequencies close to the LOS are $f_{\mathrm{b}} \in\{-433.33,433.33\} \mathrm{Hz}$ and thus a spectral width of $866.67 \mathrm{~Hz}$ is obtained directly 


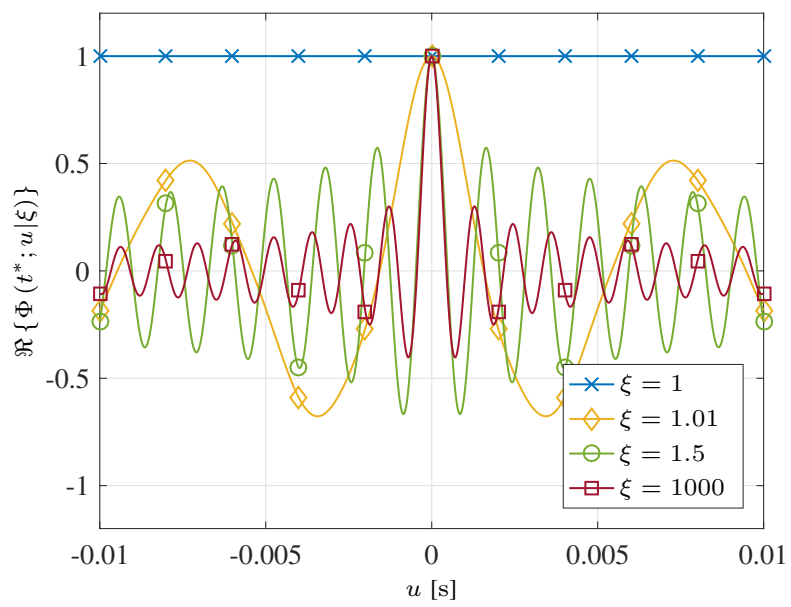

Fig. 10. Scenario 3: Real part of delay-dependent characteristic function $\Re\left\{\Phi\left(t^{*} ; u \mid \xi\right)\right\}$ for $\mathbf{v}_{\mathrm{t}}=[90,0,0]^{\mathrm{T}} \mathrm{km} / \mathrm{h}$ and $\mathbf{v}_{\mathrm{r}}=[90,0,0]^{\mathrm{T}} \mathrm{km} / \mathrm{h}$.

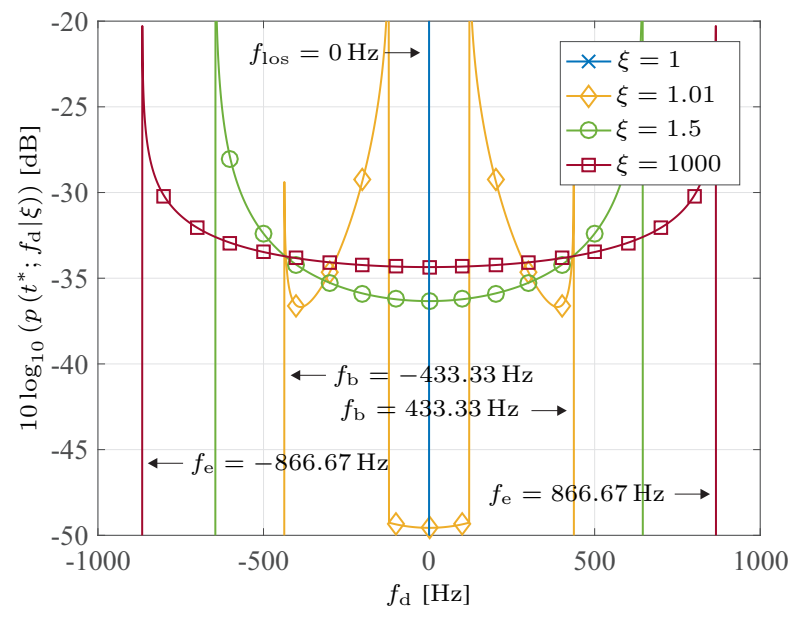

Fig. 11. Scenario 3: Delay-dependent Doppler pdf $p\left(t^{*} ; f_{\mathrm{d}} \mid \xi\right)$ and limiting frequencies $f_{\mathrm{b}}$ and $f_{\mathrm{e}}$ for $\mathbf{v}_{\mathrm{t}}=[90,0,0]^{\mathrm{T}} \mathrm{km} / \mathrm{h}$ and $\mathbf{v}_{\mathrm{r}}=[90,0,0]^{\mathrm{T}} \mathrm{km} / \mathrm{h}$.

after the LOS. For $\xi=1.01$, the influence of the LOS frequency component in the pdf is visible in Fig. 14 together with the limiting frequencies $f_{\mathrm{b}}$. The characteristic function again has roughly a triangular shape in Fig. 13. For $\xi=1.5$, the characteristic function approaches the Bessel function and the width of pdf becomes smaller as can be observed in Fig. 14. For $\xi \rightarrow \infty$, the spectral width collapses to zero, since the limiting frequency becomes $f_{\mathrm{e}}=0 \mathrm{~Hz}$. Here, the mean Doppler $\mu\left(t^{*} ; \xi\right)$ remains zero for all delays $\xi \in[0, \infty)$; the Doppler spread $\sigma\left(t^{*} ; \xi\right)$ is non-zero except $\xi \rightarrow 1$ and $\xi \rightarrow \infty$, which is shown in Fig. 15 .

The results for Scenario 5 are presented in Fig. 16, Fig. 17, and Fig. 18. The characteristic function for the LOS in Fig. 16 is a cosine function with a Doppler frequency of $f_{\text {los }}=433.33 \mathrm{~Hz}$. The limiting frequencies close to the LOS are $f_{\mathrm{b}} \in\{-433.33,0,433.33,866.67\} \mathrm{Hz}$ and thus a spectral width of $1300 \mathrm{~Hz}$ is obtained. For $\xi=1.01$, the pdf has abruptly grown and it consists of multiple parts plus the influence of the LOS frequency component, as shown in Fig. 17. The

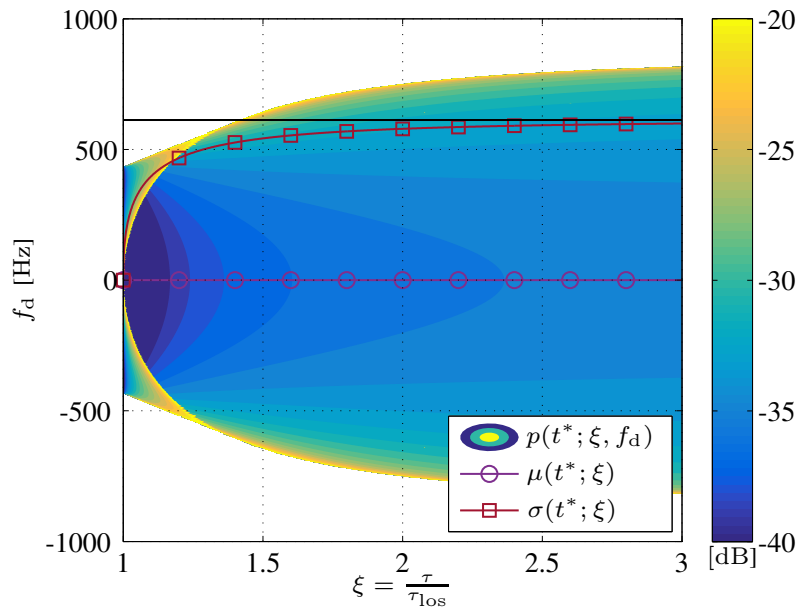

Fig. 12. Scenario 3: Joint delay Doppler pdf $p\left(t^{*} ; \xi, f_{\mathrm{d}}\right)$ with mean Doppler $\mu\left(t^{*} ; \xi\right)$, Doppler spread $\sigma\left(t^{*} ; \xi\right)$, and $\sigma$-asymptote for $\mathbf{v}_{\mathrm{t}}=$ $[90,0,0]^{\mathrm{T}} \mathrm{km} / \mathrm{h}$ and $\mathbf{v}_{\mathrm{r}}=[90,0,0]^{\mathrm{T}} \mathrm{km} / \mathrm{h}$.

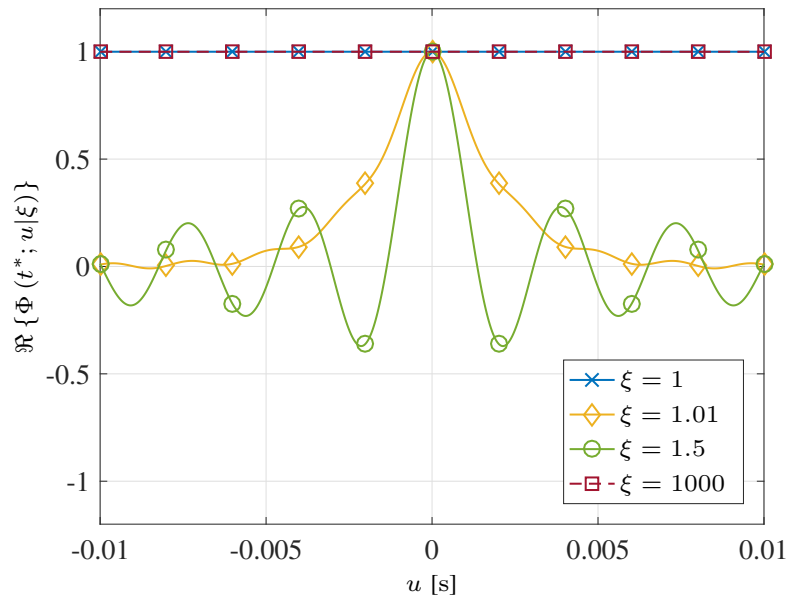

Fig. 13. Scenario 4: Real part of delay-dependent characteristic function $\Re\left\{\Phi\left(t^{*} ; u \mid \xi\right)\right\}$ for $\mathbf{v}_{\mathrm{t}}=[90,0,0]^{\mathrm{T}} \mathrm{km} / \mathrm{h}$ and $\mathbf{v}_{\mathrm{r}}=[-90,0,0]^{\mathrm{T}} \mathrm{km} / \mathrm{h}$.

spectral parts are separated by the limiting frequencies $f_{\mathrm{b}}$. The characteristic function looks like it consists of multiple functions in Fig. 16. A similar behavior is obtained for both functions as $\xi$ grows, yet with shifting limiting frequencies in the Doppler pdf. Thus, for $\xi=1.5$ the pdf consists only of two parts and the characteristic function still looks like a composition of multiple functions. From there on the limiting frequencies decrease. For $\xi \rightarrow \infty$, we obtain a Bessel function with limiting frequencies of the corresponding Doppler pdf of $f_{\mathrm{e}} \in\{-612.83,612.83\} \mathrm{Hz}$ and a spectral width of $1225.65 \mathrm{~Hz}$. The mean Doppler $\mu\left(t^{*} ; \xi\right)$ decreases monotonically, since the Doppler pdf is asymmetric and the Doppler spread $\sigma\left(t^{*} ; \xi\right)$ is monotonically increasing to its calculated asymptotic value of $\lim _{\xi \rightarrow \infty} \sigma\left(t^{*} ; \xi\right)=433.33 \mathrm{~Hz}$ in Fig. 18.

Finally, we present an exemplary visualization of the general case. The orientation of the velocity vectors of TX and RX are given in the captions of Fig. 19, Fig. 20, 


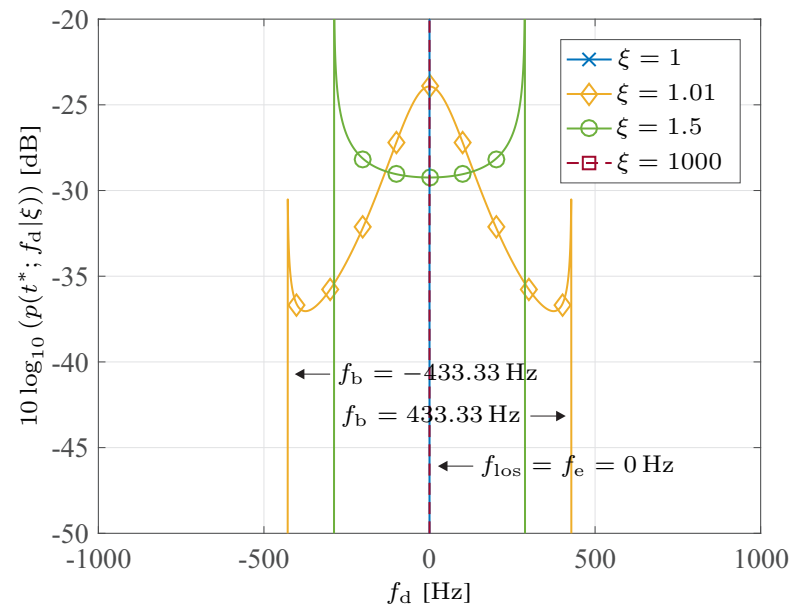

Fig. 14. Scenario 4: Delay-dependent Doppler pdf $p\left(t^{*} ; f_{\mathrm{d}} \mid \xi\right)$ and limiting frequencies $f_{\mathrm{b}}$ and $f_{\mathrm{e}}$ for $\mathbf{v}_{\mathrm{t}}=[90,0,0]^{\mathrm{T}} \mathrm{km} / \mathrm{h}$ and $\mathbf{v}_{\mathrm{r}}=$ $[-90,0,0]^{\mathrm{T}} \mathrm{km} / \mathrm{h}$.

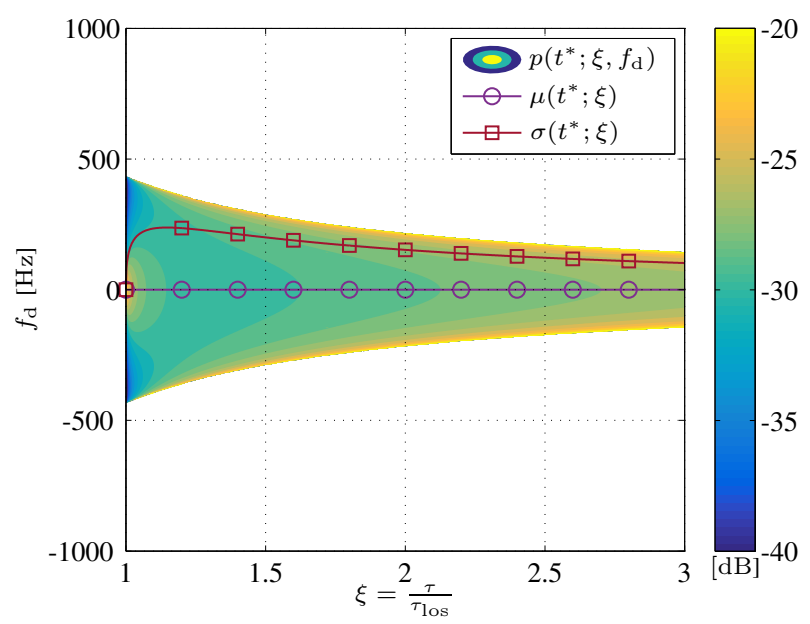

Fig. 15. Scenario 4: Joint delay Doppler pdf $p\left(t^{*} ; \xi, f_{\mathrm{d}}\right)$ with mean Doppler $\mu\left(t^{*} ; \xi\right)$, Doppler spread $\sigma\left(t^{*} ; \xi\right)$, and $\sigma$-asymptote for $\mathbf{v}_{\mathrm{t}}=$ $[90,0,0]^{\mathrm{T}} \mathrm{km} / \mathrm{h}$ and $\mathbf{v}_{\mathrm{r}}=[-90,0,0]^{\mathrm{T}} \mathrm{km} / \mathrm{h}$.

and Fig. 21. For $\xi \rightarrow 1$, the characteristic function corresponds to an exponential function, where only the velocity components along the major axis of the ellipse, i.e., along the LOS delay, contribute to the Doppler frequency, i.e., $f_{\text {los }}=-433.33 \mathrm{~Hz}$. The limiting frequencies close to the LOS are $f_{\mathrm{b}} \in\{-884.45,-665.25,306.67,376.36\} \mathrm{Hz}$. As $\xi$ increases to $\xi=1.01$, two distinctive parts and the LOS in the pdf emerge in Fig. 20. The parts are approximately bounded by the the four limiting frequencies $f_{\mathrm{b}}$ in the Fig. 20 . For $\xi=1.5$, the influence of the LOS frequency component becomes smaller and the whole pdf becomes narrower, with the two parts still visible in Fig. 20. Correspondingly, the characteristic function is similar to a composition of two Bessel functions as can be observed in Fig. 19. For $\xi \rightarrow \infty$, we obtain an exact Bessel function with corner frequencies of $f_{\mathrm{e}} \in\{-204.28,204.28\} \mathrm{Hz}$. The mean Doppler $\mu\left(t^{*} ; \xi\right)$ monotonically increases form its minimum value of $\lim _{\xi \rightarrow 1} \mu\left(t^{*} ; \xi\right)=-433.33 \mathrm{~Hz}$ to $\lim _{\xi \rightarrow \infty} \mu\left(t^{*} ; \xi\right)=0 \mathrm{~Hz}$

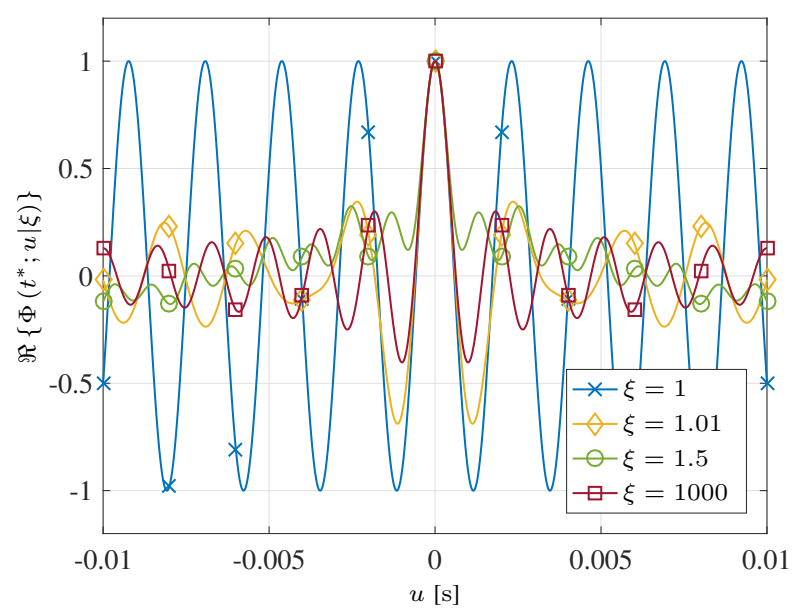

Fig. 16. Scenario 5: Real part of delay-dependent characteristic function $\Re\left\{\Phi\left(t^{*} ; u \mid \xi\right)\right\}$ for $\mathbf{v}_{\mathrm{t}}=[90,0,0]^{\mathrm{T}} \mathrm{km} / \mathrm{h}$ and $\mathbf{v}_{\mathrm{r}}=[0,0,-90]^{\mathrm{T}} \mathrm{km} / \mathrm{h}$.

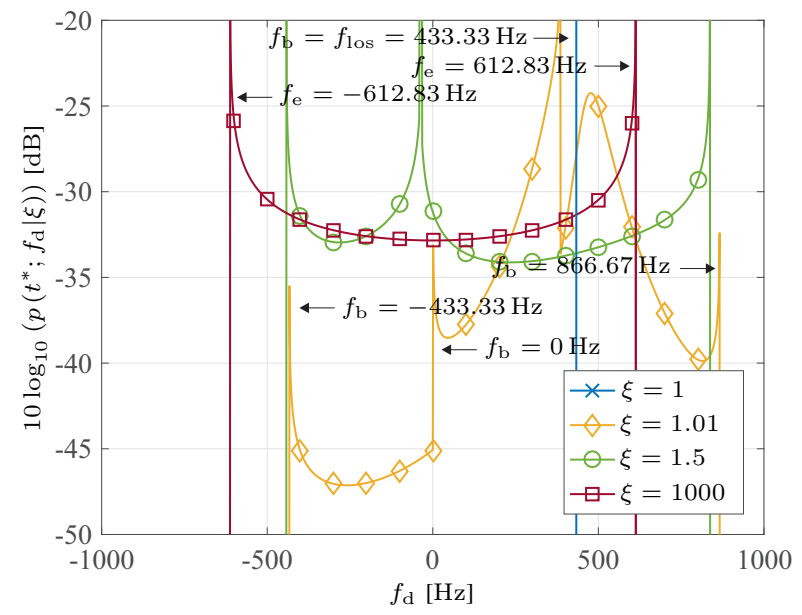

Fig. 17. Scenario 5: Delay-dependent Doppler pdf $p\left(t^{*} ; f_{\mathrm{d}} \mid \xi\right)$ and limiting frequencies $f_{\mathrm{b}}$ and $f_{\mathrm{e}}$ for $\mathbf{v}_{\mathrm{t}}=[90,0,0]^{\mathrm{T}} \mathrm{km} / \mathrm{h}$ and $\mathbf{v}_{\mathrm{r}}=$ $[0,0,-90]^{\mathrm{T}} \mathrm{km} / \mathrm{h}$.

and the Doppler spread starts at zero, reaches its maximum and then settles for $\lim _{\xi \rightarrow \infty} \sigma\left(t^{*} ; \xi\right)=144.44 \mathrm{~Hz}$ in Fig. 21 .

In comparison with the double-bounce model [8], where the correlation function consists of two Bessel functions that are multiplied with each other, the single-bounce scenario shows either an exponential or a zeroth-order Bessel function in the limiting cases, influenced by both velocity vectors. However, the components of the vectors have different influence close to the LOS and far away from it. Therefore, the width of the Doppler pdf is generally different compared to the doublebounce scenario and depends in general on the pointing of the velocity vectors and the relative delay value given by the $\xi$-coordinate.

\section{CONClusion}

With the coordinate transformation to a prolate spheroidal coordinate system, the calculation of time-variant, delaydependent characteristic functions and Doppler probability 


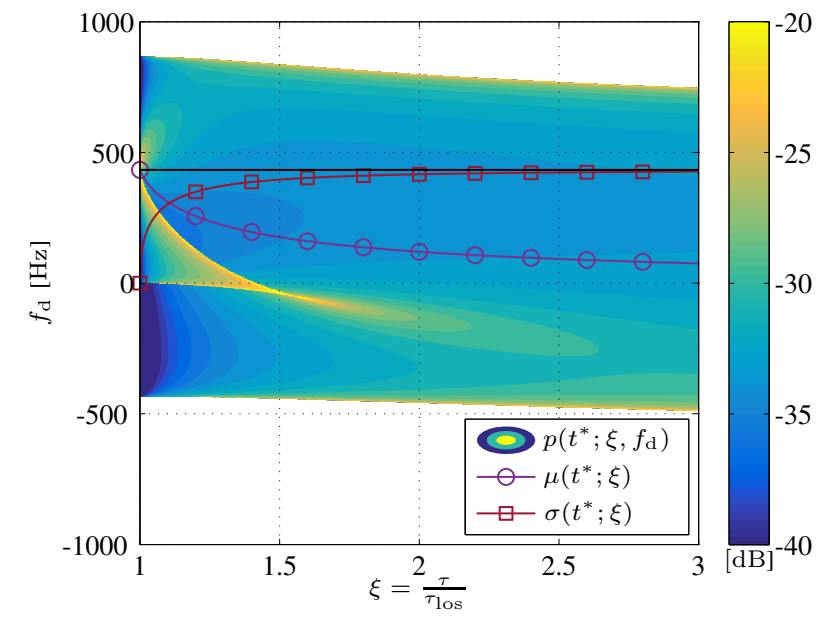

Fig. 18. Scenario 5: Joint delay Doppler pdf $p\left(t^{*} ; \xi, f_{\mathrm{d}}\right)$ with mean Doppler $\mu\left(t^{*} ; \xi\right)$, Doppler spread $\sigma\left(t^{*} ; \xi\right)$, and $\sigma$-asymptote for $\mathbf{v}_{\mathrm{t}}=$ $[90,0,0]^{\mathrm{T}} \mathrm{km} / \mathrm{h}$ and $\mathbf{v}_{\mathrm{r}}=[0,0,-90]^{\mathrm{T}} \mathrm{km} / \mathrm{h}$.

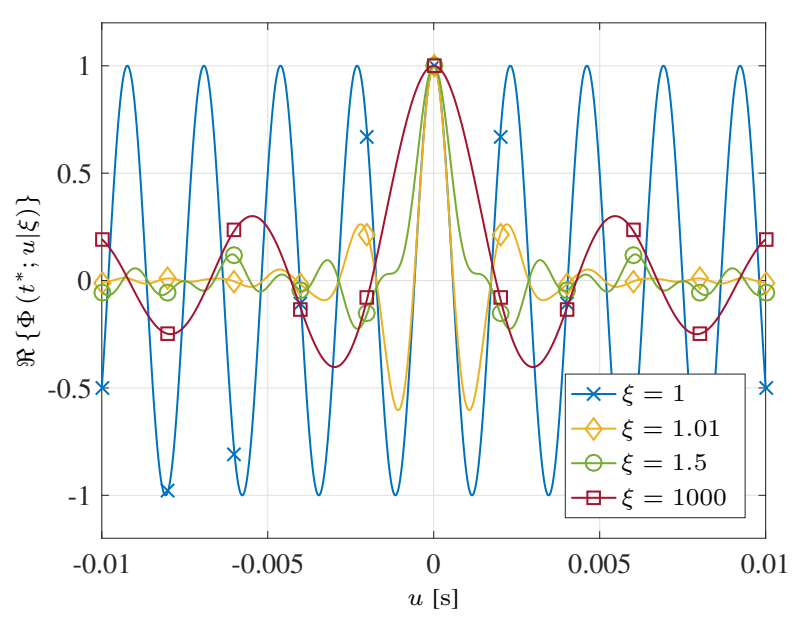

Fig. 19. Scenario 6: Real part of delay-dependent characteristic function $\Re\left\{\Phi\left(t^{*} ; u \mid \xi\right)\right\}$ for $\mathbf{v}_{\mathrm{t}}=[120,0,-30]^{\mathrm{T}} \mathrm{km} / \mathrm{h}$ and $\mathbf{v}_{\mathrm{r}}=$ $[-90,0,60]^{\mathrm{T}} \mathrm{km} / \mathrm{h}$.

density function for line-of-sight and single-bounce scattering becomes feasible for vehicle-to-vehicle channels. The investigation is based on the proportionality between probability density function and the power spectral density for nonstationary channels and a proof for this assumption has been provided. Subsequently, expressions for certain delays in typical vehicular scenarios were derived and show that line-ofsight and single-bounce case can be treated in one consistent theory. The closed-form solutions are compared to results in the literature and they are in accordance with those well-known results. Specifically, solutions for the characteristic functions contain the exponential function or the Bessel function. The Doppler probability density function becomes a Dirac delta function or the Jakes spectrum.

The abrupt transition from the zero width line-of-sight Doppler frequency to the larger-than-zero width at an $\epsilon$ vicinity of the LOS delay has been investigated. Closed-form expressions for the limiting frequencies of the Doppler pdf

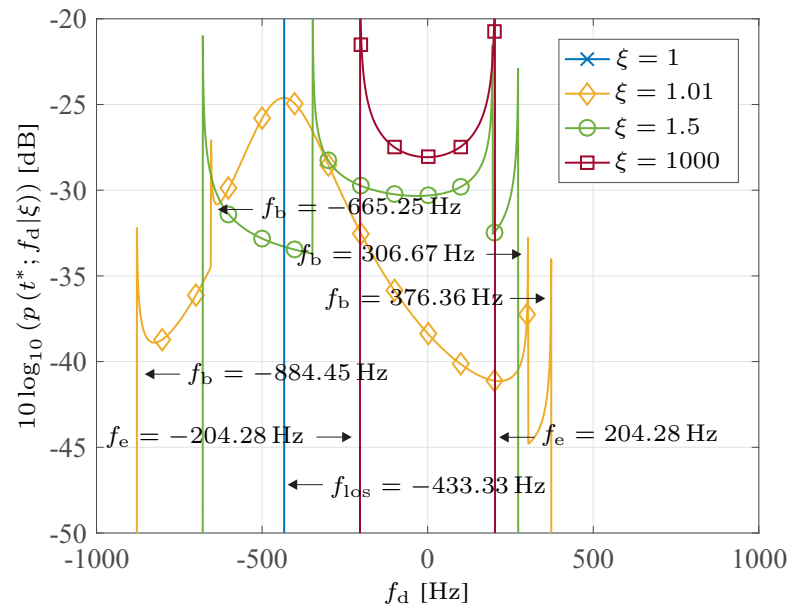

Fig. 20. Scenario 6: Delay-dependent Doppler pdf $p\left(t^{*} ; f_{\mathrm{d}} \mid \xi\right)$ and limiting frequencies $f_{\mathrm{b}}$ and $f_{\mathrm{e}}$ for $\mathbf{v}_{\mathrm{t}}=[120,0,-30]^{\mathrm{T}} \mathrm{km} / \mathrm{h}$ and $\mathbf{v}_{\mathrm{r}}=$ $[-90,0,60]^{\mathrm{T}} \mathrm{km} / \mathrm{h}$.

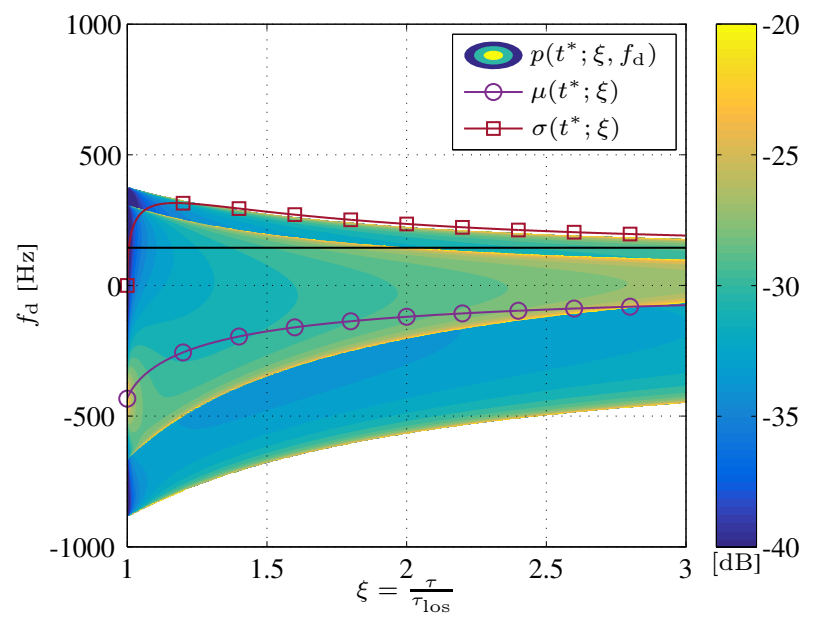

Fig. 21. Scenario 6: Joint delay Doppler pdf $p\left(t^{*} ; \xi, f_{\mathrm{d}}\right)$ with mean Doppler $\mu\left(t^{*} ; \xi\right)$, Doppler spread $\sigma\left(t^{*} ; \xi\right)$, and $\sigma$-asymptote for $\mathbf{v}_{\mathrm{t}}=$ $[120,0,-30]^{\mathrm{T}} \mathrm{km} / \mathrm{h}$ and $\mathbf{v}_{\mathrm{r}}=[-90,0,60]^{\mathrm{T}} \mathrm{km} / \mathrm{h}$.

in the $\epsilon$-vicinity of the LOS delay and for infinite delay are provided. This result is very significant, since in the $\epsilon$ vicinity of the line-of-sight the received power from scattering components is strong and therefore very important for the channel estimation.

Finally, the time-variant, delay-dependent mean Doppler and Doppler spread were directly derived from the characteristic functions. For the investigated delays, we have provided closed-form solutions, which concur well with the literature.

\section{ACKNOWLEDGMENT}

This work was partially supported by the EU project HIGHTS (High precision positioning for cooperative ITS applications) MG-3.5a-2014-636537 and the DLR project Dependable Navigation. 


\section{APPENDIX}

Please note that the velocity vectors $\mathbf{v}_{\mathrm{t}}$ and $\mathbf{v}_{\mathrm{r}}$ depend on the time $t$, yet in the following we drop the $t$ dependency for a more compact notation.

\section{A. Characteristic Functions $\Phi(t ; u \mid \xi)$ for $\xi \rightarrow 1$}

$$
\begin{aligned}
\lim _{\xi \rightarrow 1} \Phi(t ; u \mid \xi) & =\frac{1}{4} \int_{-1}^{1} \exp \left(\mathrm{j} 2 \pi u \frac{\left(v_{\mathrm{t}}^{z}-v_{\mathrm{r}}^{z}\right)}{c} f_{\mathrm{c}}\right) \mathrm{d} \eta \\
& +\frac{1}{4} \int_{-1}^{1} \exp \left(\mathrm{j} 2 \pi u \frac{\left(v_{\mathrm{t}}^{z}-v_{\mathrm{r}}^{z}\right)}{c} f_{\mathrm{c}}\right) \mathrm{d} \eta \\
& =\exp \left(\mathrm{j} 2 \pi u \frac{\left(v_{\mathrm{t}}^{z}-v_{\mathrm{r}}^{z}\right)}{c} f_{\mathrm{c}}\right) .
\end{aligned}
$$

\section{B. Characteristic Functions $\Phi(t ; u \mid \xi)$ for $\xi \rightarrow \infty$}

$\lim _{\xi \rightarrow \infty} \Phi(t ; u \mid \xi)=$

$=\int_{-1}^{1} \frac{\exp \left(\mathrm{j} 2 \pi u \frac{f_{\mathrm{c}}}{c}\left(\sqrt{1-\eta^{2}}\left(v_{\mathrm{t}}^{x}+v_{\mathrm{r}}^{x}\right)+\eta\left(v_{\mathrm{t}}^{z}+v_{\mathrm{r}}^{z}\right)\right)\right)}{2 \pi \sqrt{1-\eta^{2}}} \mathrm{~d} \eta$

$+\int_{-1}^{1} \frac{\exp \left(\mathrm{j} 2 \pi u \frac{f_{\mathrm{c}}}{c}\left(\eta\left(v_{\mathrm{t}}^{z}+v_{\mathrm{r}}^{z}\right)-\sqrt{1-\eta^{2}}\left(v_{\mathrm{t}}^{x}+v_{\mathrm{t}}^{x}\right)\right)\right)}{2 \pi \sqrt{1-\eta^{2}}} \mathrm{~d} \eta$

$=J_{0}\left(2 \pi u \frac{\left\|\mathbf{v}_{\mathrm{t}}+\mathbf{v}_{\mathbf{r}}\right\|}{c} f_{\mathrm{c}}\right)$.

C. Derivative with Respect to $u$ of Characteristic Function $\Phi(t ; u \mid \xi)$ for $\xi \rightarrow 1$

$$
\begin{aligned}
& \lim _{\xi \rightarrow 1} \frac{\partial}{\partial u} \Phi(t ; u \mid \xi)= \\
& =\frac{1}{4} \mathrm{j} 2 \pi \frac{\left(v_{\mathrm{t}}^{z}-v_{\mathrm{r}}^{z}\right)}{c} f_{\mathrm{c}} \exp \left(\mathrm{j} 2 \pi u \frac{\left(v_{\mathrm{t}}^{z}-v_{\mathrm{r}}^{z}\right)}{c} f_{\mathrm{c}}\right) \int_{-1}^{1} \mathrm{~d} \eta \\
& +\frac{1}{4} \mathrm{j} 2 \pi \frac{\left(v_{\mathrm{t}}^{z}-v_{\mathrm{r}}^{z}\right)}{c} f_{\mathrm{c}} \exp \left(\mathrm{j} 2 \pi u \frac{\left(v_{\mathrm{t}}^{z}-v_{\mathrm{r}}^{z}\right)}{c} f_{\mathrm{c}}\right) \int_{-1}^{1} \mathrm{~d} \eta \\
& =\mathrm{j} 2 \pi \frac{\left(v_{\mathrm{t}}^{z}-v_{\mathrm{r}}^{z}\right)}{c} f_{\mathrm{c}} \exp \left(\mathrm{j} 2 \pi u \frac{\left(v_{\mathrm{t}}^{z}-v_{\mathrm{r}}^{z}\right)}{c} f_{\mathrm{c}}\right) .
\end{aligned}
$$

$\lim _{\xi \rightarrow 1} \frac{\partial^{2}}{\partial u^{2}} \Phi(t ; u \mid \xi)=$

$$
\begin{aligned}
& =\frac{1}{4}\left(\mathrm{j} 2 \pi \frac{\left(v_{\mathrm{t}}^{z}-v_{\mathrm{r}}^{z}\right)}{c} f_{\mathrm{c}}\right)^{2} \exp \left(\mathrm{j} 2 \pi u \frac{\left(v_{\mathrm{t}}^{z}-v_{\mathrm{r}}^{z}\right)}{c} f_{\mathrm{c}}\right) \int_{-1}^{1} \mathrm{~d} \eta \\
& +\frac{1}{4}\left(\mathrm{j} 2 \pi \frac{\left(v_{\mathrm{t}}^{z}-v_{\mathrm{r}}^{z}\right)}{c} f_{\mathrm{c}}\right)^{2} \exp \left(\mathrm{j} 2 \pi u \frac{\left(v_{\mathrm{t}}^{z}-v_{\mathrm{r}}^{z}\right)}{c} f_{\mathrm{c}}\right) \int_{-1}^{1} \mathrm{~d} \eta . \\
& =-\left(2 \pi \frac{\left(v_{\mathrm{t}}^{z}-v_{\mathrm{r}}^{z}\right)}{c} f_{\mathrm{c}}\right)^{2} \exp \left(\mathrm{j} 2 \pi u \frac{\left(v_{\mathrm{t}}^{z}-v_{\mathrm{r}}^{z}\right)}{c} f_{\mathrm{c}}\right) .
\end{aligned}
$$

D. Derivative with Respect to $u$ of Characteristic Function $\Phi(t ; u \mid \xi)$ for $\xi \rightarrow \infty$

$$
\begin{aligned}
\lim _{\xi \rightarrow \infty} \frac{\partial}{\partial u} \Phi(t ; u \mid \xi)= \\
=\frac{1}{2 \pi} \int_{-1}^{1} \mathrm{j} 2 \pi u \frac{f_{\mathrm{c}}}{c}\left(\sqrt{1-\eta^{2}}\left(v_{\mathrm{t}}^{x}+v_{\mathrm{r}}^{x}\right)+\eta\left(v_{\mathrm{t}}^{z}+v_{\mathrm{r}}^{z}\right)\right) \\
\quad \frac{\exp \left(\mathrm{j} 2 \pi u \frac{f_{\mathrm{c}}}{c}\left(\sqrt{1-\eta^{2}}\left(v_{\mathrm{t}}^{x}+v_{\mathrm{r}}^{x}\right)+\eta\left(v_{\mathrm{t}}^{z}+v_{\mathrm{r}}^{z}\right)\right)\right)}{\sqrt{1-\eta^{2}}} \mathrm{~d} \eta \\
+\frac{1}{2 \pi} \int_{-1}^{1} \mathrm{j} 2 \pi u \frac{f_{\mathrm{c}}}{c}\left(\eta\left(v_{\mathrm{t}}^{z}+v_{\mathrm{r}}^{z}\right)-\sqrt{1-\eta^{2}}\left(v_{\mathrm{t}}^{x}+v_{\mathrm{t}}^{x}\right)\right) \\
=\frac{\exp \left(\mathrm{j} 2 \pi u \frac{f_{\mathrm{c}}}{c}\left(\eta\left(v_{\mathrm{t}}^{z}+v_{\mathrm{r}}^{z}\right)-\sqrt{1-\eta^{2}}\left(v_{\mathrm{t}}^{x}+v_{\mathrm{t}}^{x}\right)\right)\right)}{\sqrt{1-\eta^{2}}} \mathrm{~d} \eta \\
=-2 \pi \frac{\left\|\mathbf{v}_{\mathrm{t}}+\mathbf{v}_{\mathrm{r}}\right\|}{c} f_{\mathrm{c}} J_{1}\left(2 \pi u \frac{\left\|\mathbf{v}_{\mathrm{t}}+\mathbf{v}_{\mathrm{r}}\right\|}{c} f_{\mathrm{c}}\right) .
\end{aligned}
$$

$\lim _{\xi \rightarrow \infty} \frac{\partial^{2}}{\partial u^{2}} \Phi(t ; u \mid \xi)=$

$$
\begin{aligned}
= & \frac{1}{2 \pi} \int_{-1}^{1}\left(\mathrm{j} 2 \pi u \frac{f_{\mathrm{c}}}{c}\left(\sqrt{1-\eta^{2}}\left(v_{\mathrm{t}}^{x}+v_{\mathrm{r}}^{x}\right)+\eta\left(v_{\mathrm{t}}^{z}+v_{\mathrm{r}}^{z}\right)\right)\right)^{2} \\
& \frac{\exp \left(\mathrm{j} 2 \pi u \frac{f_{\mathrm{c}}}{c}\left(\sqrt{1-\eta^{2}}\left(v_{\mathrm{t}}^{x}+v_{\mathrm{r}}^{x}\right)+\eta\left(v_{\mathrm{t}}^{z}+v_{\mathrm{r}}^{z}\right)\right)\right)}{\sqrt{1-\eta^{2}}} \mathrm{~d} \eta \\
& \frac{1}{2 \pi} \int_{-1}^{1}\left(\mathrm{j} 2 \pi u \frac{f_{\mathrm{c}}}{c}\left(\eta\left(v_{\mathrm{t}}^{z}+v_{\mathrm{r}}^{z}\right)-\sqrt{1-\eta^{2}}\left(v_{\mathrm{t}}^{x}+v_{\mathrm{t}}^{x}\right)\right)\right)^{2} \\
& \frac{\exp \left(\mathrm{j} 2 \pi u \frac{f_{\mathrm{c}}}{c}\left(\eta\left(v_{\mathrm{t}}^{z}+v_{\mathrm{r}}^{z}\right)-\sqrt{1-\eta^{2}}\left(v_{\mathrm{t}}^{x}+v_{\mathrm{t}}^{x}\right)\right)\right)}{\sqrt{1-\eta^{2}}} \mathrm{~d} \eta \\
= & -\frac{1}{2}\left(2 \pi \frac{\left\|\mathbf{v}_{\mathrm{t}}+\mathbf{v}_{\mathrm{r}}\right\|}{c} f_{\mathrm{c}}\right)^{2}\left(J_{0}\left(2 \pi u \frac{\left\|\mathbf{v}_{\mathrm{t}}+\mathbf{v}_{\mathrm{r}}\right\|}{c} f_{\mathrm{c}}\right) .\right. \\
- & \left.J_{2}\left(2 \pi u \frac{\left\|\mathbf{v}_{\mathrm{t}}+\mathbf{v}_{\mathrm{r}}\right\|}{c} f_{\mathrm{c}}\right)\right) .
\end{aligned}
$$

\section{REFERENCES}

[1] K. Dar, M. Bakhouya, J. Gaber, M. Wack, and P. Lorenz, "Wireless communication technologies for its applications," IEEE Commun. Mag., vol. 48, no. 5, pp. 156-162, May 2010.

[2] R. H. Clarke, "A statistical theory of mobile-radio reception," Bell Syst. Tech. J., vol. 47, no. 6, pp. 957-1000, July/Aug. 1968.

[3] W. C. Jakes, Microwave Mobile Communications, ser. IEEE Press Classic Reissue. New York, USA: Wiley, 1994.

[4] P. A. Bello, "Characterization of randomly time-variant linear channels," IEEE Trans. Commun., vol. 11, no. 4, pp. 360-393, Dec. 1963

[5] A. Paier, T. Zemen, L. Bernadó, G. Matz, J. Karedal, N. Czink, C. Dumard, F. Tufvesson, A. F. Molisch, and C. F. Mecklenbräuker, "Non-WSSUS vehicular channel characterization in highway and urban scenarios at $5.2 \mathrm{GHz}$ using the local scattering function," in Int. ITG Workshop on Smart Antennas (WSA), Darmstadt, Germany, Feb. 2008, pp. 9-15.

[6] L. Bernadó, "Non-stationarity in vehicular wireless channels," Ph.D dissertation, Technische Universität Wien, Vienna, Austria, Apr. 2012. 
[7] G. Matz, "On non-wssus wireless fading channels," IEEE Trans. Wireless Commun., vol. 4, no. 5, pp. 2465-2478, Sept. 2005.

[8] A. S. Akki and F. Haber, "A statistical model of mobile-to-mobile land communication channel," IEEE Trans. Veh. Technol., vol. 35, no. 1, pp. 2-7, Feb. 1986.

[9] C. S. Patel, G. L. Stüber, and T. G. Pratt, "Simulation of Rayleigh-faded mobile-to-mobile communication channels," IEEE Trans. Commun., vol. 53, no. 11, pp. 1876-1884, Nov. 2005.

[10] Z. Tang and A. S. Mohan, "A correlated indoor MIMO channel model," in Canadian Conf. Elect. Comput. Eng., Montreal, QC, Canada, May 2003, pp. 1889-1892.

[11] A. G. Zajić and G. L. Stüber, "3-D MIMO mobile-to-mobile channel simulation," in 2007 16th IST Mobile and Wireless Communications Summit, Budapest, Hungary, July 2007, pp. 1-5.

[12] M. Pätzold, B. O. Hogstad, N. Youssef, and D. Kim, "A MIMO mobileto-mobile channel model: Part I - the reference model," in Proc. IEEE 16th Int. Symp. Personal, Indoor and Mobile Radio Commun., Berlin, Germany, Sept. 2005, pp. 573-578.

[13] A. Abdi and M. Kaveh, "A space-time correlation model for multielement antenna systems in mobile fading channels," IEEE J. Select. Areas Commun., vol. 20, no. 3, pp. 550-560, Apr. 2002.

[14] M. Pätzold, B. O. Hogstad, and N. Youssef, "Modeling, analysis, and simulation of MIMO mobile-to-mobile fading channels," IEEE Trans. Wireless Commun., vol. 7, no. 2, pp. 510-520, Feb. 2008.

[15] A. G. Zajić and G. L. Stüber, "Three-dimensional modeling, simulation, and capacity analysis of space-time correlated mobile-to-mobile channels," IEEE Trans. Veh. Technol., vol. 57, no. 4, pp. 2042-2054, July 2008.

[16] A. G. Zajić, G. L. Stüber, T. G. Pratt, and S. Nguyen, "Statistical modeling and experimental verification of wideband MIMO mobile-tomobile channels in highway environments," in Proc. IEEE 19th Int. Symp. Personal, Indoor and Mobile Radio Commun., Cannes, France, Sept. 2008, pp. 1-5.

[17] A. Chelli and M. Pätzold, "A non-stationary MIMO vehicle-to-vehicle channel model derived from the geometrical street model," in Proc. IEEE 74th Veh. Technol. Conf., San Francisco, CA, USA, Sept. 2011, pp. 1-6.

[18] Y. Yuan, C.-X. Wang, Y. He, M. M. Alwakeel, and e. H. M. Aggoune, "3D wideband non-stationary geometry-based stochastic models for non-isotropic MIMO vehicle-to-vehicle channels," IEEE Trans. Wireless Commun., vol. 14, no. 12, pp. 6883-6895, Dec. 2015.

[19] A. Borhani and M. Pätzold, "Correlation and spectral properties of vehicle-to-vehicle channels in the presence of moving scatterers," IEEE Trans. Veh. Technol., vol. 62, no. 9, pp. 4228-4239, Nov. 2013.

[20] A. G. Zajić, "Impact of moving scatterers on vehicle-to-vehicle narrowband channel characteristics," IEEE Trans. Veh. Technol., vol. 63, no. 7, pp. 3094-3106, Sept. 2014.

[21] M. Pätzold, Mobile Fading Channels. Chichester, UK: Wiley, 2002.

[22] M. Walter, D. Shutin, and U.-C. Fiebig, "Delay-dependent Doppler probability density functions for vehicle-to-vehicle scatter channels," IEEE Trans. Antennas Propagat., vol. 62, no. 4, pp. 2238-2249, Apr. 2014.

[23] M. Walter, U.-C. Fiebig, and A. Zajić, "Experimental verification of the non-stationary statistical model for V2V scatter channels," in Proc. IEEE 80th Veh. Technol. Conf., Vancouver, BC, Canada, Sept. 2014, pp. $1-5$.

[24] M. Walter, T. Zemen, and D. Shutin, "Empirical relationship between local scattering function and joint probability density function," in Proc. IEEE 26th Int. Symp. Personal, Indoor and Mobile Radio Commun., Hong Kong, China, Aug. 2015.

[25] J. Nie, P. A. Parrilo, and B. Sturmfels, Semidefinite Representation of the k-Ellipse. New York, NY: Springer New York, 2008, pp. 117-132.

[26] P. Hoeher, "A statistical discrete-time model for the WSSUS multipath channel," IEEE Trans. Veh. Technol., vol. 41, no. 4, pp. 461-468, Nov. 1992.

[27] M. Walter, D. Shutin, and U.-C. Fiebig, "Prolate spheroidal coordinates for modeling mobile-to-mobile channels," IEEE Antennas Wireless Propagat. Lett., vol. 14, pp. 155-158, 2015.

[28] M. Abramowitz and I. A. Stegun, Handbook of Mathematical Functions: With Formulas, Graphs, and Mathematical Tables, ser. Applied mathematics series. Dover Publications, 1965.

[29] I. N. Bronstein, K. A. Semendyayev, G. Musiol, and H. Mühlig, Handbook of Mathematics. Springer, 2007.

[30] O. Nørklit and J. B. Andersen, "Diffuse channel model and experimental results for array antennas in mobile environments," IEEE Trans. Antennas Propagat., vol. 46, no. 6, pp. 834-840, June 1998.
[31] A. Papoulis and S. U. Pillai, Probability, Random Variables, and Stochastic Processes, ser. McGraw-Hill electrical and electronic engineering series. McGraw-Hill, 2002.

[32] H. Schulze, "Stochastische Modelle und digitale Simulation von Mobilfunkkanälen," Kleinheubacher Berichte, vol. 32, pp. 473-483, 1989.

[33] R. He, O. Renaudin, V. M. Kolmonen, K. Haneda, Z. Zhong, B. Ai, and C. Oestges, "Characterization of quasi-stationarity regions for vehicleto-vehicle radio channels," IEEE Trans. Antennas Propagat., vol. 63, no. 5, pp. 2237-2251, May 2015.

[34] A. Ispas, M. Dörpinghaus, G. Ascheid, and T. Zemen, "Characterization of non-stationary channels using mismatched Wiener filtering," IEEE Trans. Signal Processing, vol. 61, no. 2, pp. 274-288, Jan. 2013.

[35] T. J. Willink, "Wide-sense stationarity of mobile MIMO radio channels," IEEE Trans. Veh. Technol., vol. 57, no. 2, pp. 704-714, Mar. 2008.

[36] P. A. Bello, "Measurement of random time-variant linear channels," IEEE Trans. Inform. Theory, vol. 15, no. 4, pp. 469-475, July 1969.

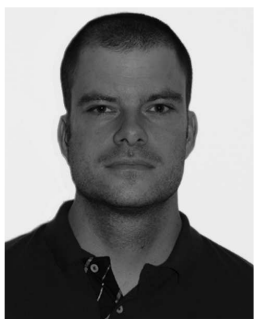

Michael Walter (M'10) received the Dipl.-Ing. (M.Sc.) and Dr.-Ing. (Ph.D.) degree in electrical engineering from Ulm University, Germany, in 2008 and 2015, respectively.

Since 2009 he has been a member of the scientific staff at the Institute of Communications and Navigation at the German Aerospace Center (DLR) in Oberpfaffenhofen, Germany, where he gained extensive experience in organizing and conducting numerous measurement campaigns for vehicular and aeronautical research. His research interests include wireless communications, modeling of mobile-to-mobile channels and channel coding.

Dr. Walter was the recipient of the IEEE/AIAA David Lubkowski Memorial for Advancement in Digital Avionics Best Paper Award of the 29th DASC in 2011.

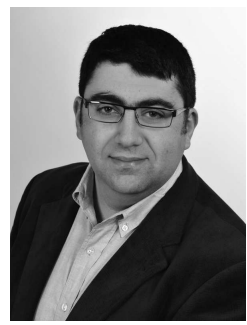

Dmitriy Shutin (M06-SM'12) received his Master degree in Computer Science in 2000 from Dniepropetrovsk State University, Ukraine, and the $\mathrm{Ph} . \mathrm{D}$. degree in electrical engineering from Graz University of Technology, Graz, Austria, in 2006.

During 2001-2006 and 2006-2009, he was a Teaching Assistant and an Assistant Professor, respectively, with the Signal Processing and Speech Communication Laboratory, Graz University of Technology. From 2009 to 2011 he was a Research Associate with the Department of Electrical Engineering at Princeton University, Princeton, NJ, USA. Since 2011 he is with the Institute of Communications and Navigation, German Aerospace Center. His current research interests include modeling and estimation of the radio propagation channels, statistical signal processing, radar signal processing, and machine learning for signal processing.

Dr. Shutin was a recipient of the Best Student Paper Award at the 2005 IEEE International Conference on Information, Communications and Signal Processing (ICICS). In 2009 he was awarded the Erwin Schroedinger Research Fellowship.

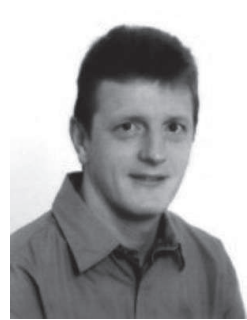

Armin Dammann received the Dipl.-Ing. (M.Sc.) and Dr.-Ing. (Ph.D.) degrees in Electrical Engineering from the University of Ulm, Germany, in 1997 and 2005 respectively. In 1997 he joined the Institute of Communications and Navigation of the German Aerospace Center (DLR). Since 2005 he is head of the Mobile Radio Transmission Research Group. His research interest and activities include signal design and signal processing for wireless communication and navigation systems. He has been active in several EU-projects, e.g., WINNER, WHERE and 